\title{
Role of the habenulo-interpeduncular system in the neurodevelopmental basis of susceptibility and resilience to stress-induced anxiety
}

Malalaniaina Rakotobe ( $\square$ malala.rakotobe08@gmail.com )

UCA iBV

Niels Fjerdingstad ( $\square$ Niels.Fjerdingstad@unice.fr )

UCA

Nuria Ruiz-Reig ( $\nabla$ nuria.ruizreig@uclouvain.be )

UCA iBV

Thomas Lamonerie ( $\nabla$ lamonerie@unice.fr)

UCA iBV

Fabien D'Autréaux ( $\nabla$ dautreaux@unice.fr )

UCA iBV https://orcid.org/0000-0002-2591-6475

Article

Keywords: habenulo-interpeduncular system, anxiety, stress, brain development

DOI: https://doi.org/10.21203/rs.3.rs-823079/v4

License: (c) (i) This work is licensed under a Creative Commons Attribution 4.0 International License.

Read Full License

Additional Declarations: There is NO Competing Interest. 


\section{2 in the neurodevelopmental basis of susceptibility and resilience to}

Malalaniaina Rakotobe ${ }^{\mathrm{a}, \S}$, Niels Fjerdingstad ${ }^{\mathrm{a}}$, Nuria Ruiz-Reig ${ }^{\mathrm{a}, \S}$, Thomas

5 Lamonerie $^{\mathrm{a}, 1,{ }^{*}}$ and Fabien D'Autréaux ${ }^{\mathrm{a}, 1, *}$.

7 France.

*Co-Corresponding authors F.D and T.L:

Emails: Fabien.D'AUTREAUX@univ-cotedazur.fr, Thomas.LAMONERIE@univ-cotedazur.fr.

Université Côte d'Azur, iBV - Institut de Biologie Valrose, Bâtiment recherche "Sciences Naturelles"

Faculté des Sciences, Parc Valrose, 06108 Nice cedex 2

${ }^{1}$ These authors contributed equally to the work.

${ }^{\S}$ The present address of M.R is at the Institut Pasteur, 75015 Paris, France and of N.R.-R, at the Institute of Neuroscience of the Universite Catholique de Louvain, 1200 Brussels, Belgium. 
Abstract

Having experienced stress during sensitive periods of brain development strongly impacts how individuals cope with later stress. Many become more prone to develop anxiety or depression, but some appear resilient. The as-yet-unknown mechanisms underlying these differences may lie in how genes and environmental stressors interact to shape the circuits controlling emotions. Here, we investigated the role of the habenulointerpeduncular system (HIPS), a critical node of reward circuits, in early stress-induced anxiety in mice. Based on immediate early gene expression, we found that a subcircuit of this system, characterized by $O t x 2$ expression, is particularly sensitive to chronic restraint stress during the peripubertal period, and that this induces hypersensitivity of the HIPS to later stress and susceptibility to develop anxiety. We also show that conditional knockout of Otx2 restricted to the HIPS in mice counteracts these effects of stress. Together, these results demonstrate that a genetic factor, $O t x 2$, and stress interact around puberty to shape the stress sensitivity of the HIPS, revealing this sensitivity as a key modulator of susceptibility/resilience to develop anxiety.

Keywords: habenula, interpeduncular nucleus, psychiatric disorders, reward circuits,

Otx2, homeobox gene, early life stress, pubertal period

\section{Introduction}

Most psychiatric illnesses occur in adolescents and adults. They are often triggered by physical or psychological trauma in susceptible individuals (Bale et al., 2010). However, most people exposed to similar traumas will not develop symptoms (Hegde and Mitra, 2020). Understanding how susceptibility or resilience to psychiatric disorders is established is therefore an important health issue. Susceptibility or resilience may have a neurodevelopmental 
specific sensitive periods of development (Assary et al., 2018; Lopatina et al., 2021). Key contributing environmental factors include chronic stresses experienced early in life, which are predisposing factors for depression and anxiety (Ross et al., 2017) and are widely used in animal models to identify the circuits and mechanisms involved. Among validated forms of chronic stress (CS) inducing anxiety-like and depression-related behaviors in rodents, restraint is very effective (Chiba et al., 2012) (Seewoo et al., 2020). This painless procedure induces a form a psychological stress, does not have the confounding effects of other physical stresses and can be applied at any stage of postnatal development including pre- and post-weaning. The developmental stages to which stress is applied, is indeed another critical parameter. Depending on it, different processes may be affected, and behavioral outcomes may vary considerably. Regarding anxiety and depression, the risk of their occurrence during adolescence and adulthood is increased by stresses endured during the peripubertal period (Holder and Blaustein, 2014; Horovitz et al., 2012; Winer et al., 2016).

Among brain circuits, the reward system is a prime candidate to be the substrate of stressinduced anxiety-like and depression-like behaviors. Indeed, there is a strong connectivity between stress response and reward circuits and, in fact, considerable overlap between the two (Bouarab et al., 2019; Metzger et al., 2021). Recently recognized as a component of reward circuits, the habenulo-interpeduncular system (HIPS), is also involved in the regulation of mood, novelty attraction, motivation, and memory (McLaughlin et al., 2017; Viswanath et al., 2013). This system is composed of the medial habenula (MHb) and its unique output, the interpeduncular nucleus (IPN). There is growing evidence that the HIPS plays a critical role in feedback from cortical and subcortical regions to the brainstem to elicit adapted emotional responses. Clinical and animal models studies link HIPS dysfunction to mood disorders 
hypothesis, developmental changes in the HIPS during periods when this circuit is plastic could be at the origin of these disorders.

While the plasticity of other components of reward circuits, such as the amygdala and the ventral tegmental area (VTA), in response to early adversity has begun to be studied (Birnie et al., 2020; Pena et al., 2017), research on the effect of early stress on HIPS plasticity and function is still scarce. These studies are primarily focusing on its physiology in adults (Lee et al., 2019; Vickstrom et al., 2020; Yoo et al., 2021), and the few studies on HIPS development have not addressed the direct relationship between HIPS developmental abnormalities and stress-induced pathologies (Aizawa et al., 2011; Hsu et al., 2016). Furthermore, the HIPS is involved in many aspects of behavioral control and is known to contain heterogenous neuronal populations with different developmental origins, identities and outputs (Lorente-Canovas et al., 2012; Quina et al., 2017; Ruiz-Reig et al., 2019). However, which function is associated with which population is unclear.

We recently characterized a circuit within the HIPS (Ruiz-Reig et al., 2019), which requires Otx2 to develop normally. The habenular neurons of this subcircuit are in the most medial part of both the dorsal $\mathrm{MHb}(\mathrm{dMHb})$ and ventral $\mathrm{MHb}(\mathrm{vMHb})$ with highest expression of Otx2 $\left(\mathrm{Otx} 2^{\mathrm{High}}\right)$. They preferentially target regions of the IPN that contain Otx $2^{+}$neurons, with which they contact synaptically. Importantly, habenular and IPN neurons in this circuit retain Otx2 expression throughout development and adulthood. We named this subcircuit the HIPOPS (Habenulo-InterPeduncular-Otx2-Positive-System). Otx2 is a developmental gene that encodes a homeoprotein with important brain functions during development and in adulthood (Beby and Lamonerie, 2013; Gat-Yablonski, 2011). Otx2 is highly conserved throughout evolution

81 (Ranade et al., 2008; Sen et al., 2013), making it a good marker of conserved brain circuits.

82 Based on the known functions of Otx2 and the HIPS, we hypothesize that the HIPOPS plays important and conserved functions inside the reward system. 
Otx2 marks several other interconnected brain regions that also belong to the reward circuits.

For example, Otx2 plays a major role during specific critical periods of VTA postnatal development (Pena et al., 2017) reducing its expression through Early life stress (ELS) induces long-term susceptibility to depression-like behavior. Thus, Otx2 is an important player in critical periods of neuronal development and modulating its expression can lead to long-term effects on the reward system. The fact that Otx2 is linked to human pathologies involving the reward system reinforces this idea. Indeed, it is a susceptibility gene for bipolar diseases and stress-related disorders (Maheu and Ressler, 2017; Sabunciyan et al., 2007). Because HIPS dysfunctions are also involved in these diseases, Otx2 roles in critical periods of HIPOPS development may be important in this process.

In this study, we developed a new paradigm to investigate the neurodevelopmental basis of risk/resistance to specific psychiatric disorders linked to the HIPOPS. We used restraint, as a stressor. We set up a two-hit restraint protocol in which the first stress applied during puberty is meant to generate susceptibility or resilience that translate into a pathological state only following a second period or "hit" of stress later (Daskalakis et al., 2013). To demonstrate the sensitivity of the HIPOPS to stress, rather than direct recording of electrical activity, we measured the expression of immediate early genes that integrate more cellular activities. For instance, Egrl is regulated by a wide variety of environmental stimuli, including stress, and may in turn regulate the transcriptional program related to neuronal activity and synaptic plasticity (Gallo et al., 2018). Furthermore, Egrl is associated with neuropsychiatric disorders in which neuronal plasticity and activity are altered, such as stress-induced anxiety. Using our stress paradigm and monitoring Egrl activation, we have highlighted the existence of a critical period of HIPOPS development under the influence of environmental stressors. Individuals subjected to restraint stress during this period exhibit specific HIPOPS hyperactivity relative to other circuits of the HIPS. These changes do not in themselves cause an anxiety state, but 
induce long-term changes in the circuitry that make animals more prone to develop anxiety-

110 like behaviors. Furthermore, we demonstrate that $O t x 2$ is a contributing factor since reducing

111 its expression specifically in the HIPOPS alters the stress response and abolishes this long-term

112 susceptibility.

\section{2. Material and methods}

114 2.1. Animals

115 All mice used were generated and maintained in the animal house of the Institut de Biologie

116 Valrose, Nice, France. All mouse lines were kept in the 129/Sv background. Only males were

117 used in this study to avoid having effects due to sexual dimorphism regarding sexual maturation

118 period and stress response. Otx $2^{\text {CreERT2/+ }}$ and Otx $2^{\text {floxflox }}$ mice were generated as described

119 previously (Fossat et al., 2006). The $\mathrm{Ngn1}^{\mathrm{CreERT2/+}}$;Ail4/Ai14 line was kindly provided by Lisa

120 V. Goodrich (Koundakjian et al., 2007). The Ai14 allele (Gt(ROSA)26Sor(tdTomato-WPRE)

121 was used as the reporter of $\mathrm{Ngn} 1^{\mathrm{CreERT} /+}$ expression. Adult mice were used for viral injection

122 of DREADDs. Behavioral tests were done during the day cycle of the $12 \mathrm{~h}$ day/night cycle.

123 Postnatal deletion Otx2 was achieved by injecting intraperitoneally $30 \mu 1$ of tamoxifen in

124 neonates at $\mathrm{P} 0$ at a concentration of $2,5 \mathrm{mg} / \mathrm{ml}$ with $300 \mu \mathrm{l}$ insulin syringes. Brains from all the

125 mice that went through behavioral testing were analyzed post-mortem and showed successful

126 recombination. Care and handling of the animals prior or during the experimental procedures

127 followed European Union rules and were approved by the French Animal Care and local ethic

128 Committees.

2.2. DREADDs microinjections

130 Animals were anesthetized by intraperitoneal injection (IP injection) with a mix of Tiletamine-

131 Buprenorphine-Zolazepam-Xylazine (TBZX) $(60 \mathrm{mg} / \mathrm{kg})$. Xylocaïne gel was applied on the

132 skull prior to the incision and in the ear to lessen the pain perception. After disinfection of the 
area of incision, each mouse was successively placed and maintained on the stereotaxic

134 apparatus. The eyes were protected from drying with ocry-gel. The cannula was implanted unilaterally from the bregma based on Paxinos et Franklin (2004). The implantation was

136 successively made on the left and then on the right side, in the $\mathrm{MHb}$ according to these

137 coordinates : $\mathrm{A} / \mathrm{P}=-1,5 ; \mathrm{D} / \mathrm{V}=-2,75$; lateral $=+/-0,2.500 \mathrm{nl}$ of the activating Gq- coupled 138 DREADDs hM3D receptor (pAAV9-hSyn-DIO-hM3D(Gq)-mCherry, $100 \mu \mathrm{L}$ from titer $\geq$ $1391 \times 10^{13} \mathrm{vg} / \mathrm{mL}$ ) (Cat No. 44361-AAV9; Addgene, USA) were injected at a speed of $100 \mathrm{nl} / \mathrm{min}$ 140 in $\mathrm{Otx} 2^{\mathrm{CreERT2/+}}$ or $\mathrm{Otx} 2^{\text {floxfllox }}$ and $\mathrm{Ot} x 2^{+/+}$control mice bilaterally. At the end of the injection,

141 the cannula was left in place for infusion for $5 \mathrm{~min}$ at the site of injection before withdrawal.

142 At the end of the surgery, an anti-inflammatory (Metacam; ,5 mg/ml) was administered 143 intraperitoneally. The anti-inflammatory treatment was repeated for 3 days post-surgery. Ten 144 days after the transduction, tamoxifen (Sigma-Aldrich) diluted in corn oil at a concentration of $14510 \mathrm{mg} / \mathrm{ml}$ was IP injected at $5 \mu \mathrm{l} / \mathrm{g}$ of body weight to induce the translocation into the nucleus 146 of CreERT2 and, thus, the excision of the STOP-cassette that was preventing the expression of $147 \mathrm{hM} 3 \mathrm{D}$ in the neurons of the MHb. Ten days after the tamoxifen injection, Clozapine $\mathrm{N}$-oxide 148 hydrochloride (CNO) (SML2304, Sigma-Aldrich) diluted in DMSO was IP injected at a 149 concentration of $0,3 \mathrm{mg} / \mathrm{kg}$ to activate hM3D signaling pathway and thus depolarization of the 150 neurons. The mice were left undisturbed, for $7 \mathrm{~h}$, to let Egr1 expression return to baseline, as it 151 is induced by the stress of the injection, and then they were rapidly sacrificed.

\subsection{Stress protocol}

Mice were placed in pierced syringes suited for their size at each stage studied, to restrain their movements as much as possible. The restraint was used acutely (one unique $2 \mathrm{~h}$-restraint) or 155 chronically ( $2 \mathrm{~h} /$ day for 7 days). For the analysis related to the study of neuronal activity at the 156 different developmental stages, the mice were immediately sacrificed at the end of the acute stress or at the end of the last chronic stress. The control mice that did not undergo any protocol 
of stress were quickly sacrificed at the proper age. At the end of the behavioral analysis, mice for each condition were divided into two groups: a group of mice that underwent a last restraint stress before the collection of the brains, and a group that was immediately sacrificed.

\subsection{Chronic sucrose consumption}

162 Food pellet containing 40\% sucrose was given every day for 6 days. Mice were sacrificed the 163 last day, 2 hours after consumption of the pellet.

The open field test and the elevated plus maze test were used to assess anxiety level during the Behavior 1 and Behavior 2 steps. We also assessed the avoidance of a new object level at both steps. Depression level was assessed using the forced swim test.

Mice were acclimated in the behavioral room $1 \mathrm{~h}$ prior to the beginning of the tests. Videos were acquired and analyzed with the EthoVision XfT software. For all the tests, the arena was cleaned with $70 \%$ ethanol and dried at the end of each trial.

172 The open field test was used to measure the anxiety levels and the ambulatory behaviors of the mice. The test was recorded in an arena in which the walls and the floor are made of black PVC. The box dimension is (40 W x $40 \mathrm{~L} \times 30 \mathrm{H} \mathrm{cm})$ and was divided into the border area

175 (50\% of the total arena) and into the center area $\left(800 \mathrm{~cm}^{2}\right)$. The field was moderately illuminated at 60 lux. Mice were individually placed in a corner of the arena and the behavior was recorded for $5 \mathrm{~min}$. The time spent, the latency to enter, the number of entries, the mean speed and the total distance travelled in the center, which is an anxiogenic area for the mice, were measured. 
181 The Elevated Plus Maze test (EPM test) was used to assess the anxiety level. This apparatus is

$18250 \mathrm{~cm}$ elevated from the ground and composed of the open arms $(50 \times 5 \mathrm{~cm})$ which are

183 anxiogenic for the mice, and two closed arms (50 W x $5 \mathrm{~L}$ x $16 \mathrm{H} \mathrm{cm})$ which spread from the

184 central platform $(5 \times 5 \mathrm{~cm})$. Mice were placed at the center of the platform, head toward a

185 closed arm and the video was recorded for $5 \mathrm{~min}$. The latency to explore the open arms and the

186 time spent in the open arms were measured.

This test is based on the conflict between exploratory and avoidance behavior in the presence of a new object (Powell et al., 2004; Roy and Chapillon, 2004). Excessive avoidance is the hallmark of anxiety disorders (Pittig et al., 2018). An exploratory zone, containing a transparent cylinder with holes, was designed in the middle of one side of the wall of the open field. Mice were placed facing that side and the video was recorded for 5 min (Habituation phase).

193 Following the habituation phase, an object was placed inside the cylinder and the mouse was relocated at the opposite side of the object and the video was recorded for 5 more min (Exploration of the object phase). The percentage of time spent in the exploratory zone, during the exploration of the object phase, is calculated and the statistics were made on the values obtained. We also used these values to divide the mice into subgroups: mice that spent a percentage of time $\leq 40 \%$ in the exploratory zone are classified as avoiding/anxious, those that spend a percentage of time $\geq 60 \%$ are classified as exploratory/curious, the mice in between are classified as indifferent.

\subsection{Forced swim test}

202 The forced swim test was used to measure the depression level of mice. Mice were placed individually in a transparent cylinder $(12 \mathrm{~cm}$ diameter; $30 \mathrm{~cm}$ of height) high enough to avoid 
min. The time of immobility during the last 4 min was measured on the EthoVision software.

206 The immobility was defined as the lack of movement, except those necessary to sustain the

207 head outside of the water. The water was changed at each trial.

2.10. Immunohistochemistry and histological analysis

209 Mice used to identify critical periods of development were directly sacrificed at the end of the

210 acute stress or at the end of the last stress in the two-hit stress paradigm. Brains were dissected

211 and directly frozen in Tissue-Tek OCT compound at $-80^{\circ} \mathrm{C}$ (Fisher Scientific, Waltham, MA,

212 USA). In order to preserve endogenous fluorescence of the reporter, mice transduced with the

213 DREADDs were subjected to intra-cardiac perfusion using PBS, then $4 \%$ paraformaldehyde

214 (PFA). The same treatment was done to Otx $^{+/+} ; \mathrm{Ngn1}^{\mathrm{CreERT2/+}} ; \mathrm{Ai14/Ai14}$, Otx $2^{\text {floxflox }} ; \mathrm{Ngn1}^{\mathrm{CreERT2/+}} ;$ Ail4/Ai14 and Otx $2^{\text {floxflox }}$ Ail4/Ail4 mice used to study the

216 anatomical effect of Otx2 deletion in the $\mathrm{MHb}$, the neuronal activity in response to stress

217 between P30-36 and to mice that underwent the behavioral analysis. Brains were post-fixed overnight in $4 \%$ PFA at $4^{\circ} \mathrm{C}$ and cryoprotected in $30 \%$ PBS sucrose before freezing in TissueTek OCT compound at $-80^{\circ} \mathrm{C}$. Eyes were collected from dislocated mice and placed in cold PBS. A small hole was done at the level of the ora serrata. The eyes were fixed in 4\% PFA for $2 \mathrm{~h}$ at room temperature, cryoprotected in $20 \%$ PBS sucrose overnight, then frozen in OCT and stored at $-80^{\circ} \mathrm{C}$.

Brains were cut in $16 \mu \mathrm{m}$ coronal sections using a Microm HM550 cryostat and sections were mounted on SuperFrost+ slides (Fisher Scientific). Eyes were cut in $14 \mu \mathrm{m}$ sections collected on SuperFrost+ slides. The sections directly frozen were post-fixed in fresh $4 \%$ PFA. Before 226 the blocking step, sections from perfused $\mathrm{Otx}^{+/+} ; \mathrm{Ngn1}^{\mathrm{CreERT2/+}} ; \mathrm{Ai14} / \mathrm{Ai14}$, Otx $2^{\text {flox/flox }} ; \mathrm{Ngn1}^{\mathrm{CreERT2/+}} ; \mathrm{Ai14/Ai14}$ and $\mathrm{Otx} 2^{\text {floxfflox }}$ Ai14/Ail4 mice were subjected to an unmasking step to better reveal the Otx 2 signal that is weaker in perfused brains. In this step, 
the slides were incubated in Citrate Buffer, $\mathrm{pH} 6$, for 8 min at $95^{\circ} \mathrm{C}$. A second treatment was performed with PBS-Glycine (100 mM glycine) to limit background noise. The rest of the steps

231 were similar for all the samples. Briefly, the slides were incubated in a blocking solution (PBS

232 with $0,2 \%$ dTriton $10 \%$ FBS) for 1 h. The primary antibodies were incubated overnight at $4{ }^{\circ} \mathrm{C}$ 233 in a solution of PBS $(0,1 \%$ Triton- $10 \%$ FBS $)$. The sections were incubated 10 min in DAPI $234(1 \mu \mathrm{g} / \mathrm{ml})$.

235 The primary antibodies used were: mouse anti-Brn3a (Merck/Millipore, \#MAB1585, 1:250), 236 rabbit anti-Er81 (kind donation of Silvia Arber, 1:1000), goat anti-Otx (R\&D Systems, 237 \#AF1979, 1 :500), rabbit anti c-fos (SySy, \#226004 1 :2000), rabbit anti-egr1 (cell signaling, $238 \quad \# 15 F 7,1$ :500), guinea-pig anti-Parvalbumin (SySy, \#195004, 1 :500).

239 The secondary antibodies used (Jackson ImmunoResearch, 1:500) were: anti-rabbit Alexa 240 Fluor $^{\mathrm{TM}}$ 488, anti-rabbit Alexa Fluor ${ }^{\mathrm{TM}}$ 647, anti-goat Cy3, anti-mouse Alexa Fluor ${ }^{\mathrm{TM}}$ 647, 241 anti-guinea-pig Cy3.

2.11. Microscopy and immunoreactivity measures Images of the retina were acquired on a confocal microscope Zeiss 780 (Zeiss, Oberkochen, 244 Germany). All other images were acquired on the wide-field microscope AxioObserver - Zeiss 245 (2011) with the sCMOS ANDOR Neo camera and the stereomicroscope coupled with the digital camera (Zeiss axioplan2). Images were processed with the ImageJ software and figures were mounted on Adobe Photoshop. For Egr1 immunoreactivity measurements, the mouse brain atlas, fourth edition was used to delineate the different regions. For the medial habenula,

2498 sections of $16 \mu \mathrm{m}$ thick per animal were selected every 5 sections that covered bregma $-1,22$ 250 to $-1,94 \mathrm{~mm}$. Both sides were analyzed and averaged. For the caudal IPN, 3 sections of $16 \mu \mathrm{m}$ thick per animal were selected every 5 sections that covered bregma $-3,4$ to $-3,88 \mathrm{~mm}$, covering the entire Otx2+ area of the IPN. The area of Otx2+ neurons was specifically delineated for the 
analysis of Egr1 immunoreactivity in Otx2+ neurons. All medial habenula sections at all the stages and for all conditions were analyzed together and the same was done for all the interpeduncular nucleus sections considered. Analysis was made using imageJ plugins "Analyze particles" with a thresholding that was identical for all conditions.

\subsection{Statistical analysis}

Statistical analyses were made using the R software. Comparison was made within the same genotypes and between different stress conditions. Results are presented as the mean \pm SEM. The comparison of the mean of two groups was done by using the Student t-test when the variance was equal and with a Welch correction when it was not. Comparison of three groups were made with a one-way ANOVA. The two parameters, genotype and stress condition, were compared with a two-way ANOVA, using ANOVA function of the package "car" and corrected for the type III error for unbalanced datasets. Statistical details can be found in figure legends and in supplemental materials (Supplemental table S1). To study the effect on anxiety levels of the interaction between stress and genotype, mean ranking was computed as follows. A rank was attributed to the mice according to the value of the variable (for example time spent in the open arms of the EPM), ordered increasingly among all mice independently of their genotype or stress condition. Global scoring of anxiety corresponded to the mean scoring between all parameters (time, distance, and number of entry) from both tests (EPM and Open Field).

\section{Results} activity 
278 Previous results showed that MHb neurons express Otx2 following a gradient (Ruiz-Reig et

$279 a l ., 2019)$. All neurons located in the Otx $2^{\text {High }}$ region maintain high levels of Otx2 till 280 adulthood. Otx $2^{\text {High }}$ neurons preferentially make synapses with Otx $2^{+}$IPN neurons, all located 281 in the caudal IPN (cIPN), suggesting that Otx2 marks a subcircuit inside the HIPS that may be 282 preferentially interconnected (Ruiz-Reig et al., 2019). To test this hypothesis further, we asked 283 whether chemogenetic activation of $\mathrm{MHb}$ Otx $2^{\text {High }}$ neurons that are glutamatergic would 284 preferentially drive the activation of their targets in the cIPN of mice. We forced activation of 285 Otx $2^{\text {high }} \mathrm{MHb}$ neurons in $\mathrm{Otx} 2^{\mathrm{CreERT2/+}}$ mice through a DREADD-hM3D-approach (Fig1a), 286 consisting of CreERT2-dependent expression and CNO-dependent activation of the 287 DREADDs, and monitored neuronal activation (Fig1b) by examining the expression of 288 immediate-early genes (IEGs), strongly and specifically induced after neuronal activation 289 (Zangenehpour and Chaudhuri, 2002). The approach was specific because in tamoxifen290 injected control mice that did not express the CreERT2 $\left(\right.$ Ot $\left.x 2^{+/+}\right)$or that expressed the 291 CreERT2 $\left(\right.$ Otx $\left.2^{\mathrm{CreERT2/+}}\right)$ but did not receive CNO, no evidence of neuronal activation could be 292 detected in the MHb and IPN (Supplemental Fig.S1 and graph Fig.1). In contrast, upon tamoxifen and CNO injections in $\mathrm{Otx} 2^{\mathrm{CrEERT2/+}}$ mice, a strong Fos-immunoreactivity was found

294 in $\mathrm{dMHb}$ neurons expressing hM3D in the Otx $2^{\mathrm{High}}$ region (Fig.1d-f). In cIPN targets, neuronal 295 activity could be detected in the two Otx $2^{+}$subnuclei, the med-cIPN (medio-caudal IPN) and 296 lat-cIPN (latero-caudal IPN) (Fig.1g,h). Moreover, the large majority of cells strongly Fos297 immunoreactive were surrounded by hM3D-mCherry and expressed Otx2 (86 $\pm 8 \%$ of total 298 Fos-immunoreactive cells) (Fig.1h1-h2' and Graph Fig.1).

299 Thus, activation of Otx $2^{\text {High }}$ neurons in the $\mathrm{MHb}$ triggers activity mainly in Otx $2^{+}$neurons of 300 the IPN, confirming that these neurons form a sub-circuit within the HIPS that can be marked 301 by the expression of Otx2, the HIPOPS. 
305 We then investigated the response of habenular neurons and their IPN targets in mice facing stress stimuli. To explore different developmental stages (P14-20, P30-P37, P37-P43 or P6066), we selected physical restraint, which is the only type of stress that can be applied identically at any stage. We tested the effect of a chronic stress (CS) on HIPS activity by applying the same restraint stress 2 hours a day for 7 consecutive days, on male mice, and

310 looking at IEGs immediately after the last stress. Expression of c-Fos and Egrl (Krox-24, Zif-

311 268), another IEG (Zangenehpour and Chaudhuri, 2002), was strongly induced in the dMHb

312 compared to control in Otx $2^{\text {High }}$ neurons (Fig.2a-f). Egr1 appeared an even more robust marker 313 of stress-induced activity (Fig.2c,d versus Fig.2c',d') and was used thereafter. Egr1 induction 314 was restricted to Otx $2^{+}$neurons (Fig.2e,f) and was statistically stronger than controls only post315 weaning into the pre- (P30-36) and adolescence (P37-43) stages (Fig.2c-d', graph Fig.2j and 316 Supplemental Fig.S2), but not before weaning or in the adult (P14-20 and P60-66, graph

317 Fig.2j). Following the same trend, activation of the IPN was statistically stronger than controls 318 at the same stages (Fig.2h-i', graph Fig.2k). The level of induction of Egr1 expression was 319 increased in both Otx2+ and Otx2- neurons (Graph Fig.21). The number of Otx2+ and Otx2neurons expressing Egr1 tended to increase and reached significance for Otx2+ neurons (Graph Fig.2m). The ratio between CS and NS conditions for Otx2+ and Otx2- populations revealed that Otx2+ neurons, upon CS, were preferentially induced based on the level of Egr1immunoreactivity while the number of induced neurons were similar (graph Fig.2n-o). In the

324 lat-cIPN, 2 different Otx $2^{+}$populations could be distinguished based on their precise location 325 and molecular profile. Only L-lat-cIPN neurons strongly expressed parvalbumin and were 326 surrounded by a perineuronal net (PNN), a specialized extracellular matrix marked by the lectin 
327 WFA (Supplemental Fig.S3). Furthermore, only Otx2+ neurons of the med- and M-lat-cIPN, 328 but not of the L-lat-cIPN, were strongly activated by CS (Supplemental Fig.S4). We then tested 329 the effect of an acute stress (AS) of 2 hrs restraint, immediately after, which was unable to 330 activate neurons of the MHb (Supplemental Fig.S5a-c). In the med- and M-lat-cIPN, activity 331 of Otx2+ neurons was not different from a non-stressed condition, whereas, it was statistically 332 different in the L-lat-cIPN (Supplemental Fig.S5d-f, graph Fig.S5g). Accordingly, the ratio "stress/no stress" was statistically different between AS and CS conditions (Supplemental graph Fig.S5h).

To verify that this activation was due to stress and not to any other type of event, we tested the response of the HIPOPS following a chronic exposure to a pleasurable event such as pleasanttasting food with high content of sucrose. This condition elicited no detectable response (Supplemental Fig.S3f).

Thus, while AS does not activate the $\mathrm{MHb}$ and involves Otx $2^{+} / \mathrm{PV}^{+}$neurons in the IPN, the

340 HIPOPS specifically responds to CS. Repeated restraint activates MHb neurons in the Otx $2^{\text {high }}$ 341 region and consequently, Otx $2^{+}$neurons in the med-cIPN and M-lat-cIPN are preferentially 342 induced. The peak of activation is around puberty (P30-P36). adult stress We then examined the consequences of CS applied during puberty on the susceptibility

348 to develop anxiety-like behavior later. We used a two-hit stress model, composed of a mild stress (same stress used above of 2 hours restraint for 7 consecutive days) during the HIPOPS 
hyper-responsive period (P30-36), to generate the susceptibility, followed by a second hit of

351 CS in the adult, to reveal this susceptibility at the behavioral level (Fig.3a).

352 Mice subjected to CS (P30-36) were tested for anxiety levels 2 weeks after the end of the stress.

353 The behavior of the stressed mice was indistinguishable from that of the control mice (Fig.3bg; "controls") in two independent behavioral tests known to detect anxiety-like behavior in rodent, the open-field and the elevated plus maze tests (EPM), demonstrating the absence of a direct pathological consequence of the first stress. In sharp contrast, several weeks after a second period of stress applied during early adulthood (P60-P66), animals that also had received the early stress presented statistically higher anxiety-like behavior, based on distance travelled, time spent and number of entries in the open-field, and based on distance travelled and time spent in the open arm of the EPM (Fig.4a-c,e "Controls"). Difference in number of entries in the EPM did not reach significance, although it followed the same trend. Global scoring considering all the parameters of both tests (see material and methods) confirmed a statistical difference (Fig. $4 \mathrm{j}$ "Controls"). These differences were not due to motor dysfunctions

364 (Fig.4g-h "Controls"). Depressive-like behavior was also not significantly affected (Fig.4i "Controls"). Interestingly, avoidance of a novel object was increased (Fig.4k), in line with their increased anxiety in the other tests.

367 These results show for the first time that a CS, composed of repeated restraint sessions during puberty and able to strongly activate the HIPOPS, induces susceptibility to anxiety-like behavior, only revealed at the adult stage upon stressful events. This time window may thus represent a critical period of HIPOPS development involved in setting long-term anxiety levels. anxiety-like behavior on the long-term. 
375 To directly implicate the HIPOPS in the mechanisms leading to anxiety-like behavior, we

376 tested whether interfering with the normal development of the HIPOPS would change the

377 outcome of individuals experiencing the early stress. Since Otx2 is a master gene of HIPOPS

378 development, we used the $N g n 1^{\text {CreERT2 }}$ line to delete Otx2 specifically in this circuit (Kim et al.,

379 2011; Koundakjian et al., 2007). In this strain, CreERT2 expression becomes gradually

380 restricted as mice age and overlaps Otx2 expressing sites only in the dMHb and rare

381 photoreceptors in the retina, as shown by the lineage of CreERT2 expressing cells followed

382 from P0 to P30 (Supplemental Fig.S6 and FigS7).

383 Otx2 deletion was triggered by tamoxifen injection at P0. To be able to compare the 384 experimental groups, all animals received tamoxifen. At P30, Otx $2^{\text {floxflox }} ;$ Ngn $^{\text {CreERT2 }}$ animals

385 were compared to tamoxifen-treated $O t x 2^{f l o x f f l o x}$ or to $O t x 2^{+/+} ; N g n 1^{\text {CreERT2 }}$ mice. The habenula

386 in these mice appeared normal. Projections from the neurons that had lost $\operatorname{Otx} 2$ expression were

387 maintained, surrounding their IPN targets (Supplemental Fig.S6b-e'). The size of the MHb was

388 unchanged further supporting lack of structural abnormalities in the circuit (Supplemental

389 Fig.S6f). We next assessed the behavior of these mice at the adult stage of P100. No

390 abnormalities were detected in term of anxiety levels or motor parameters such as mean speed

391 or distance travelled in the open field (Supplemental Fig.S8a-b).

392 Thus, CreERT2 expression or postnatal Otx2 deletion in the dMHb and in rare cells in the retina does not result in anatomical or behavioral abnormalities under standard conditions.

394 Because the $\mathrm{dMHb}$ is particularly activated by CS, especially during the critical period of

395 HIPOPS development (Fig.2), and because the HIPOPS is closely dependent on Otx2 gene

396 function, we tested whether Otx2 deletion would affect stress-induced anxiety-like behavior.

397 We first assessed behavior after an initial stress hit. As in control mice, we did not detect 
398 behavioral abnormalities induced by the first stress (Fig.3b-g "cKO"). We next analyzed 399 behaviors after the two-hit stress protocol. As expected, in both controls, the two-hit stress 400 protocol increased anxiety levels; in contrast, this effect of stress could not be observed in $401 O t x 2^{\text {floxfllox }} ; \mathrm{Ngn1} 1^{\text {CreERT2 }}$ mice (Supplemental Fig.S8f). In fact, all the parameters that were 402 affected in control animals, after the early + late stress compared to the late stress alone, were 403 not affected in $O t x 2^{\text {floxflox}} ; N g n 1^{\text {CreERT2 }}$ mice (Fig.4a-j). Comparison of the global anxiety level 404 score confirmed that only control mice were statistically affected by the two-Hit stress protocol

405 (Fig.4j). The time of inactivity in the FST test was similar in $O t x 2^{\text {floxfflox }}$;Ngn1 ${ }^{\text {CreERT2 }}$ mice with 406 or without the early stress (Fig.4i), as expected, since the stress-protocol does not induce 407 depressive-like symptoms in controls. Avoidance of a novel object test revealed that the 408 proportion of individuals avoiding, exploring or indifferent to a new object was similar between cKO animals without stress or with the two-hit stress protocol (Fig.41). This result is different from what we observed in control animals (Fig.4k). Interestingly, the effect of adult stress alone

411 followed the same trend as in the controls with an increase in the proportion of mice indifferent 412 or avoiding the new object (Fig.4k,l; middle histogram), and the time spent in the 413 exploratory/curious zone (Fig.4m).

414 Thus, deletion of $O t x 2$ in the dMHb results in a relative protection against the effects of stress 415 on the long-term regarding anxiety and avoidance of a new object. This resilience effect 416 appears to be the consequence of an altered $\mathrm{G} \times \mathrm{E}$ interaction, between Otx2 and stress 417 specifically during the juvenile period.

3.5. Deletion of Otx2 restricted to the HIPOPS leads to a bimodal response to juvenile chronic stress and protects from stress-hypersensitivity of the circuit later on. 
422 To understand the mechanisms of resilience to stress induced by Otx2 ablation in the dMHb, 423 we analyzed the HIPOPS response to juvenile stress in $O t x 2^{\text {floxflox }} ; \mathrm{Ngn1}{ }^{\text {CreERT2 }}$ animals (Fig.5).

424 Interestingly, in these mice, although the $\mathrm{dMHb}$ was even more active than in controls 425 following juvenile CS (Fig.5a-b), this did not induce any activation of IPN neurons (Fig.5c-d). 426 This might suggest that Otx2 deletion results in abnormal connectivity between the dMHb and 427 its IPN output. However, the strong activation of Otx $2^{+}$med-cIPN and M-lat-cIPN neurons by an acute stress (AS) (Fig.5c2-c2'), in correlation with a strong activation of the dMHb (Fig.5a2a2') at P30, ruled out this hypothesis. This was all the more surprising because an AS normally does not significantly activate the dMHb at all time points tested (Fig.5a1-a1', c1-c1' and supplemental Fig.S5 and S9).

432 Thus, deletion of $\operatorname{Otx} 2$ in $O t x 2^{\text {floxfllox }} ; \mathrm{Ngn} 1^{\mathrm{CreERT2}}$ mice exacerbates the stress sensitivity of the habenular part of the HIPOPS circuit. This habenular hyper-sensitivity, paradoxically, renders

434 IPN neurons insensitive after 5-7 days of stress.

435 We then tested whether this biphasic response to the early stress would have an impact on longterm response of the circuit to stress. We examined the status of the circuit at the adult stage (P130), in control and Otx2 cKO mice that had or had not undergone the two-hit protocol, when subjected to a final AS. No activity was detected in the $\mathrm{dMHb}$ in any of the conditions except rare cells in the $\mathrm{dMHb}$ of control animals with previous two-hit stress (Supplemental

440 Fig.S10a1,b1,c1). There was tendency of higher activity in IPN neurons in control animals with previous two-hit stress compared to all other conditions that only reach significance compared to the one in Otx2 cKO with prior two-hit stress (Fig.5e-g). Activity in Otx2 cKO appeared surprisingly low, particularly in the L-lat-cIPN, where $\mathrm{PNN}^{+}$neurons are readily activated even

444 in control animals without previous two-hit stress (Fig.5f1-f2'). Interestingly, many other 445 regions known to be involved in stress response followed the same pattern as the HIPOPS. 446 Indeed, regions such as the paraventricular nucleus of the thalamus (PV) or the LHb (Reviewed 
in(Hikosaka, 2010; Rowson and Pleil, 2021) and supplemental Fig.S10) presented a higher activity in control mice than in $O t x 2 \mathrm{cKO}$ animals subjected to previous two-hit stress.

Otx2 deletion in the $\mathrm{dMHb}$ thus changes the normal HIPOPS response to a biphasic response with hyperactivation at the onset of stress followed by insensitivity of the IPN targets as stress becomes chronic. In the long term, Otx2 ablation in the $\mathrm{dMHb}$ protects against the development of stress hyperreactivity in HIPOPS neurons and in stress-related circuits, correlating with apparent protection against anxiety.

\section{Discussion}

We showed that a CS but not an AS nor pleasurable stimuli during the preadolescent (P30-36) and early adolescent (P37-43) periods causes a strong activation of the $\mathrm{dMHb}$ that correlates with a strong activation of Otx2 $2^{+}$IPN neurons in the med-cIPN and M-lat-cIPN. This correlation supports a direct activation of IPN neurons by the MHb. Indeed, we showed that Otx $2^{\text {High }}$ neurons ((Ruiz-Reig et al., 2018) and this study) project specifically and directly to Otx $2^{+}$neurons of the IPN. Furthermore, DREADD-mediated activation of Otx $2^{\text {High }}$ neurons, specifically triggers activity of Otx $2^{+}$IPN neurons and only is those that are surrounded by

463 DREADD ${ }^{+}$fibers in the med-and lat-cIPN. In animals with a specific deletion of Otx2 in the $\mathrm{dMHb}$, there is a relative stronger activation of Otx $2^{\mathrm{High}}$ habenular neurons by a stressful experience, correlated with a paradoxical insensitivity of Otx $2^{+}$IPN neurons towards the end of the stress period that may be due to long term depression upon overstimulation of the IPN or a gradual loss of function of Otx $2^{\text {high }}$ neurons, leading to stress hypo-response of Otx $2^{+}$IPN neurons, in adults. Plasticity mechanisms has been shown recently between $\mathrm{dMHb}$ and LatcIPN neurons regulated through opposing actions of glycinergic and substance $\mathrm{P}$ transmission 
471 of the juvenile stress correlates with the activity observed on the long term, suggesting that stress experiences at this juvenile period sets the intensity of IPN response to further stress episodes. Puberty may therefore represent a critical period of HIPOPS development when environmental factors such as stress interact with genes of development to shape IPN functions involved in the control of anxiety (Supplemental Fig.S11). This could explain why individuals depending on their trauma history and genetic background develop resistance rather that susceptibility to disease. Lower levels of Otx2 in the HIPOPS could be an important factor in promoting resilience, at least at these developmental periods. This fits well with the hypoanxiety that has been recently described in Otx2 heterozygote mice in which Otx 2 expression is twofold lower (Vincent et al., 2021). This study and ours would seem contradictory to what was described in the VTA where Otx2 deletion mimicks stress effect (Pena et al., 2017), however, in that latter study the brain circuits and the critical period considered is different and depression-like symptoms are triggered. Furthermore, Otx2 expression is only transiently decreased which may have very different effect on neuronal activity. In any case, collectively these results and many others (Bernard and Prochiantz, 2016) show that Otx2 is involved in critical periods of development, and from our study in the opening and closure of a critical period of HIPOPS development regarding stress response. The protection against stressinduced anxiety observed in Otx2 cKO mice cannot be explained by the few recombined photoreceptors. Indeed, deletion of $O t x 2$ in all photoreceptors does not affect anxiety levels (Pensieri et al., 2021). Furthermore, the effect of the deletion in only revealed when combined with stress and only during HIPOPS critical period of stress response.

Roles of Otx2 during the critical periods include the control of the maturation of $\mathrm{PV}^{+}$cells through non-cell autonomous mechanisms that involves the binding of Otx2 to PNNs surrounding these neurons (Vincent et al., 2021). In this study we found that the L-lat-cIPN is enriched in cells that are strongly $\mathrm{PV}^{+}$and surrounded by PNNs revealed by their high content 
in glycoconjugates that bind to Wisteria floribunda agglutinin (WFA). These Otx $2^{+}$neurons

497 are induced by acute but not CS as opposed to other Otx $2^{+}$neurons in the IPN (M-lat-cIPN and

498 med-cIPN) and Otx $2^{\text {high }}$ neurons of the MHb. It is thus possible that a low activity of the MHb

499 upon AS (below our detection threshold) is able to induce L-lat-cIPN neurons only, whereas a

500 stronger activity is required to induce the other populations. This difference of response may

501 be linked to the presence of PNN structures, only surrounding L-lat-cIPN, that may increase synaptic efficiency. If this is the case, it is surprising that high activity of the MHb upon CS, is not followed by high activity of L-lat-cIPN. An inhibitory mechanism upon CS may thus

504 prevent these cells to respond to MHb neurons. Alternatively, response to acute stress may be

505 less dependent on habenular activity, although these cells are known to receive and to respond 506 to habenular inputs (Melani et al., 2019). Interestingly, we found that CS during the critical 507 period leads to an abnormal response to AS on the long term. Anxious-like mice are characterized by high activity of the cIPN whereas resistant mice present an abnormally low activity of the cIPN. Knowing the important role of PNNs in restricting plasticity associated

510 with learning and that PNN maturation is influenced by physiological stimuli, such as stress

511 (Pesarico et al., 2019), CS-induced activity of the MHb may thus modify PNN structures 512 leading to increased or decreased stress-response of the HIPOPS in controls or Otx2 cKO

513 respectively. It is also tempting to speculate that a non-cell autonomous mechanism of Otx2

514 operates here in the maturation and function of $\mathrm{PV}^{+}$cells, as described in other systems

515 (Vincent et al., 2021), and that the source of extracellular Otx2 comes from the MHb

516 projections. The reduction of Otx 2 in $\mathrm{cKO}$ animals may thus alter the maturation of PV+ cells

517 and PNNs. However, in contrast to other systems described so far, L-lat-cIPN neurons express

518 Otx2 (Ruiz-Reig et al., 2018) so they may not require external sources of Otx2, unless

519 endogenous and exogenous Otx2 have different functions. 
520 To monitor HIPOPS response to stress we relied on the expression of immediate early genes such as Egrl and c-Fos (see introduction). Whether or not they reflected direct neuronal activity, the changes in Egrl levels we found are significant and may be even more valuable to assess specific changes of the cells that may have gone unnoticed or difficult to find by direct recording. Nevertheless, a thorough electrophysiological study on brain slices in vitro would certainly help to understand which electrophysiological phenotype correlates with the change of Egr1 expression.

In conclusion, we have shown that chronic restraint stress, during puberty, specifically induces the HIPOPS circuit within the HIPS and makes animals more prone to develop high levels of anxiety but not depression. At the circuit level, high anxiety is correlated to stress hypersensitivity of the HIPOPS and of the whole stress-related circuit. These neuronal and behavioral effects must be driven through the HIPOPS because $O t x 2$ deletion restricted to the $\mathrm{dMHb}$ interferes with juvenile stress only and renders the animals less prone to develop anxiety. This demonstrates the existence of a G x E interaction at the HIPOPS level between genetic factors, such as Otx2, and CS, during a critical period of development. Clinical data point to puberty as, a window of vulnerability to anxiety, depression, schizophrenia, and substance abuse (Dahl, 2004; Patton and Viner, 2007), and exposure to stress plays an important role (Grant et al., 2004). It is therefore of utmost importance to further investigate

538 the role of the HIPOPS during this period as it may hold the key to understand susceptibility

539 versus resilience to stress-induced anxiety in human. The two-hit stress protocol that we used 540 is a valuable tool to address this question. Indeed, one can study neuronal circuit abnormalities 541 responsible for susceptibility or resilience without confounding effects of a pathological state 542 only triggered after the second hit of stress, which would be extremely difficult to observe in 543 clinic. Also, having previously shown that Otx2 is important for HIPOPS early development, 544 we now suggest that Otx2 is a master gene of HIPOPS maturation and function. Also known 
as a susceptibility gene for psychiatric disorders in human, Otx2 may therefore act as such through the HIPOPS.

Author contributions: F.D wrote the first draft of the paper. T.L, M.R and F.D wrote the paper. M.R and F.D designed the research. N.R.-R, N.F, M.R and F.D performed the research. T.L contributed unpublished reagents/tools and most of the financial support. T.L, M.R, N.F and F.D analyzed the data.

Aknowledgements: We would like to particularly thank Benjamin Lapijover and Erwann Touzet for preliminary data, Aurélie Biancardini for mouse husbandry, Mouna Sekoni

Khemiri for genotyping. We would like to thank Olivier Deschaux and Michèle Studer for sharing behavioral equipment and software. We would like to thank Lisa V. Goodrich for the Ngn1 ${ }^{\text {CreERT2 }}$ line and Silvia Arber for the Er81 antibodies. We would like to thank the iBV PRISM and Histo-pathology platforms and especially Simon Lachambre and Samah Rekima.

Funding sources: This work was supported by the University of Nice (CSI fundings,

561 Programme de Recherches Avancées UCA ${ }^{\mathrm{JEDI}}$ ), the National Center for Scientific Research 562 (CNRS), the French ministry of Research (MESR fellowship).

Conflict of interest: The authors of this manuscript declare no competing financial interests.

Preprint Servers: This article has been deposited as a preprint on research square

567 platform. All preprints on the platform are published under a CC-BY 4.0 license. 
Aizawa, H., Amo, R., Okamoto, H., 2011. Phylogeny and ontogeny of the habenular structure.

Front Neurosci 5, 138.
Assary, E., Vincent, J.P., Keers, R., Pluess, M., 2018. Gene-environment interaction and psychiatric disorders: Review and future directions. Semin Cell Dev Biol 77, 133-143.

Bale, T.L., Baram, T.Z., Brown, A.S., Goldstein, J.M., Insel, T.R., McCarthy, M.M., Nemeroff, C.B., Reyes, T.M., Simerly, R.B., Susser, E.S., Nestler, E.J., 2010. Early life programming and neurodevelopmental disorders. Biol Psychiatry 68, 314-319.

Beby, F., Lamonerie, T., 2013. The homeobox gene Otx2 in development and disease. Exp Eye Res 111, 9-16.

Bernard, C., Prochiantz, A., 2016. Otx2-PNN Interaction to Regulate Cortical Plasticity. Neural Plast 2016, 7931693.

Birnie, M.T., Kooiker, C.L., Short, A.K., Bolton, J.L., Chen, Y., Baram, T.Z., 2020. Plasticity of the Reward Circuitry After Early-Life Adversity: Mechanisms and Significance. Biol Psychiatry 87, 875-884.

Bouarab, C., Thompson, B., Polter, A.M., 2019. VTA GABA Neurons at the Interface of Stress and Reward. Frontiers in neural circuits 13, 78.

Chiba, S., Numakawa, T., Ninomiya, M., Richards, M.C., Wakabayashi, C., Kunugi, H., 2012. Chronic restraint stress causes anxiety- and depression-like behaviors, downregulates glucocorticoid receptor expression, and attenuates glutamate release induced by brainderived neurotrophic factor in the prefrontal cortex. Prog Neuropsychopharmacol Biol Psychiatry 39, 112-119.

Dahl, R.E., 2004. Adolescent brain development: a period of vulnerabilities and opportunities. Keynote address. Ann N Y Acad Sci 1021, 1-22.

Daskalakis, N.P., Bagot, R.C., Parker, K.J., Vinkers, C.H., de Kloet, E.R., 2013. The three-hit concept of vulnerability and resilience: toward understanding adaptation to early-life adversity outcome. Psychoneuroendocrinology 38, 1858-1873.

Fossat, N., Chatelain, G., Brun, G., Lamonerie, T., 2006. Temporal and spatial delineation of mouse Otx2 functions by conditional self-knockout. EMBO Rep 7, 824-830.

Gallo, F.T., Katche, C., Morici, J.F., Medina, J.H., Weisstaub, N.V., 2018. Immediate Early Genes, Memory and Psychiatric Disorders: Focus on c-Fos, Egr1 and Arc. Front Behav Neurosci 12, 79.

Gat-Yablonski, G., 2011. Brain development is a multi-level regulated process--the case of the OTX2 gene. Pediatr Endocrinol Rev 9, 422-430.

Grant, K.E., Compas, B.E., Thurm, A.E., McMahon, S.D., Gipson, P.Y., 2004. Stressors and child and adolescent psychopathology: measurement issues and prospective effects. $J$ Clin Child Adolesc Psychol 33, 412-425.

Hegde, A., Mitra, R., 2020. Environment and early life: Decisive factors for stress-resilience and vulnerability. Int Rev Neurobiol 150, 155-185.

Hikosaka, O., 2010. The habenula: from stress evasion to value-based decision-making. Nat Rev Neurosci 11, 503-513.

Holder, M.K., Blaustein, J.D., 2014. Puberty and adolescence as a time of vulnerability to stressors that alter neurobehavioral processes. Front Neuroendocrinol 35, 89-110.

Horovitz, O., Tsoory, M.M., Hall, J., Jacobson-Pick, S., Richter-Levin, G., 2012. Post-weaning to pre-pubertal ('juvenile') stress: a model of induced predisposition to stress-related disorders. Neuroendocrinology 95, 56-64. 
Hsu, Y.W., Morton, G., Guy, E.G., Wang, S.D., Turner, E.E., 2016. Dorsal Medial Habenula Regulation of Mood-Related Behaviors and Primary Reinforcement by TachykininExpressing Habenula Neurons. eNeuro 3.

Kim, E.J., Hori, K., Wyckoff, A., Dickel, L.K., Koundakjian, E.J., Goodrich, L.V., Johnson, J.E., 2011. Spatiotemporal fate map of neurogenin1 (Neurog1) lineages in the mouse central nervous system. J Comp Neurol 519, 1355-1370.

Koundakjian, E.J., Appler, J.L., Goodrich, L.V., 2007. Auditory neurons make stereotyped wiring decisions before maturation of their targets. $J$ Neurosci 27, 14078-14088.

Lee, H.W., Yang, S.H., Kim, J.Y., Kim, H., 2019. The Role of the Medial Habenula Cholinergic System in Addiction and Emotion-Associated Behaviors. Front Psychiatry 10,100 .

Lopatina, O.L., Panina, Y.A., Malinovskaya, N.A., Salmina, A.B., 2021. Early life stress and brain plasticity: from molecular alterations to aberrant memory and behavior. Rev Neurosci 32, 131-142.

Lorente-Canovas, B., Marin, F., Corral-San-Miguel, R., Hidalgo-Sanchez, M., Ferran, J.L., Puelles, L., Aroca, P., 2012. Multiple origins, migratory paths and molecular profiles of cells populating the avian interpeduncular nucleus. Dev Biol 361, 12-26.

Maheu, M.E., Ressler, K.J., 2017. Developmental pathway genes and neural plasticity underlying emotional learning and stress-related disorders. Learn Mem 24, 492-501.

McLaughlin, I., Dani, J.A., De Biasi, M., 2017. The medial habenula and interpeduncular nucleus circuitry is critical in addiction, anxiety, and mood regulation. $J$ Neurochem 142 Suppl 2, 130-143.

Melani, R., Von Itter, R., Jing, D., Koppensteiner, P., Ninan, I., 2019. Opposing effects of an atypical glycinergic and substance $\mathrm{P}$ transmission on interpeduncular nucleus plasticity. Neuropsychopharmacology 44, 1828-1836.

Metzger, M., Souza, R., Lima, L.B., Bueno, D., Goncalves, L., Sego, C., Donato, J., Jr., Shammah-Lagnado, S.J., 2021. Habenular connections with the dopaminergic and serotonergic system and their role in stress-related psychiatric disorders. Eur J Neurosci $53,65-88$.

Patton, G.C., Viner, R., 2007. Pubertal transitions in health. Lancet 369, 1130-1139.

Pena, C.J., Kronman, H.G., Walker, D.M., Cates, H.M., Bagot, R.C., Purushothaman, I., Issler, O., Loh, Y.E., Leong, T., Kiraly, D.D., Goodman, E., Neve, R.L., Shen, L., Nestler, E.J., 2017. Early life stress confers lifelong stress susceptibility in mice via ventral tegmental area OTX2. Science 356, 1185-1188.

Pensieri, P., Mantilleri, A., Plassard, D., Furukawa, T., Moya, K.L., Prochiantz, A., Lamonerie, T., 2021. Photoreceptor cKO of OTX2 Enhances OTX2 Intercellular Transfer in the Retina and Causes Photophobia. eNeuro 8.

Pesarico, A.P., Bueno-Fernandez, C., Guirado, R., Gomez-Climent, M.A., Curto, Y., Carceller, H., Nacher, J., 2019. Chronic Stress Modulates Interneuronal Plasticity: Effects on PSA-NCAM and Perineuronal Nets in Cortical and Extracortical Regions. Front Cell Neurosci 13, 197.

Pittig, A., Treanor, M., LeBeau, R.T., Craske, M.G., 2018. The role of associative fear and avoidance learning in anxiety disorders: Gaps and directions for future research. Neurosci Biobehav Rev 88, 117-140.

Powell, S.B., Geyer, M.A., Gallagher, D., Paulus, M.P., 2004. The balance between approach and avoidance behaviors in a novel object exploration paradigm in mice. Behavioural Brain Research 152, 341-349.

Quina, L.A., Harris, J., Zeng, H., Turner, E.E., 2017. Specific connections of the interpeduncular subnuclei reveal distinct components of the habenulopeduncular pathway. J Comp Neurol 525, 2632-2656. 
682

683

684

685

686

687

688

689

690

691

692

693

694

695

696

697

698

699

700

701

702

703

704

Ranade, S.S., Yang-Zhou, D., Kong, S.W., McDonald, E.C., Cook, T.A., Pignoni, F., 2008. Analysis of the Otd-dependent transcriptome supports the evolutionary conservation of CRX/OTX/OTD functions in flies and vertebrates. Dev Biol 315, 521-534.

Ranft, K., Dobrowolny, H., Krell, D., Bielau, H., Bogerts, B., Bernstein, H.G., 2010. Evidence for structural abnormalities of the human habenular complex in affective disorders but not in schizophrenia. Psychol Med 40, 557-567.

Ross, R.A., Foster, S.L., Ionescu, D.F., 2017. The Role of Chronic Stress in Anxious Depression. Chronic Stress (Thousand Oaks) 1, 2470547016689472.

Rowson, S.A., Pleil, K.E., 2021. Influences of Stress and Sex on the Paraventricular Thalamus: Implications for Motivated Behavior. Front Behav Neurosci 15, 636203.

Roy, V., Chapillon, P., 2004. Further evidences that risk assessment and object exploration behaviours are useful to evaluate emotional reactivity in rodents. Behav Brain Res 154, 439-448.

Ruiz-Reig, N., Rakotobe, M., Bethus, I., le Menn, G., Huditz, H.I., Marie, H., Lamonerie, T., D'Autreaux, F., 2018. Developmental requirement of homeoprotein Otx2 for specific habenulo-interpeduncular subcircuits. J Neurosci.

Ruiz-Reig, N., Rakotobe, M., Bethus, I., Le Menn, G., Huditz, H.I., Marie, H., Lamonerie, T., D'Autreaux, F., 2019. Developmental Requirement of Homeoprotein Otx2 for Specific Habenulo-Interpeduncular Subcircuits. J Neurosci 39, 1005-1019.

Sabunciyan, S., Yolken, R., Ragan, C.M., Potash, J.B., Nimgaonkar, V.L., Dickerson, F., Llenos, I.C., Weis, S., 2007. Polymorphisms in the homeobox gene OTX2 may be a risk factor for bipolar disorder. Am J Med Genet B Neuropsychiatr Genet 144B, 10831086.

Seewoo, B.J., Hennessy, L.A., Feindel, K.W., Etherington, S.J., Croarkin, P.E., Rodger, J., 2020. Validation of Chronic Restraint Stress Model in Young Adult Rats for the Study of Depression Using Longitudinal Multimodal MR Imaging. eNeuro 7.

Sen, S., Reichert, H., VijayRaghavan, K., 2013. Conserved roles of ems/Emx and otd/Otx genes in olfactory and visual system development in Drosophila and mouse. Open Biol 3, 120177.

Vickstrom, C.R., Liu, X., Liu, S., Hu, M.M., Mu, L., Hu, Y., Yu, H., Love, S.L., Hillard, C.J., Liu, Q.S., 2020. Role of endocannabinoid signaling in a septohabenular pathway in the regulation of anxiety- and depressive-like behavior. Mol Psychiatry.

Vincent, C., Gilabert-Juan, J., Gibel-Russo, R., Alvarez-Fischer, D., Krebs, M.O., Le Pen, G., Prochiantz, A., Di Nardo, A.A., 2021. Non-cell-autonomous OTX2 transcription factor regulates anxiety-related behavior in the mouse. Mol Psychiatry.

Viswanath, H., Carter, A.Q., Baldwin, P.R., Molfese, D.L., Salas, R., 2013. The medial habenula: still neglected. Front Hum Neurosci 7, 931.

Winer, J.P., Parent, J., Forehand, R., Breslend, N.L., 2016. Interactive Effects of Psychosocial Stress and Early Pubertal Timing on Youth Depression and Anxiety: Contextual Amplification in Family and Peer Environments. J Child Fam Stud 25, 1375-1384.

Yoo, H., Yang, S.H., Kim, J.Y., Yang, E., Park, H.S., Lee, S.J., Rhyu, I.J., Turecki, G., Lee, H.W., Kim, H., 2021. Down-regulation of habenular calcium-dependent secretion activator 2 induces despair-like behavior. Scientific reports 11, 3700.

Zangenehpour, S., Chaudhuri, A., 2002. Differential induction and decay curves of c-fos and zif268 revealed through dual activity maps. Brain Res Mol Brain Res 109, 221-225. 


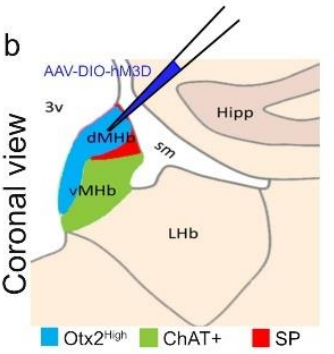

C Projection map caudal IPN level
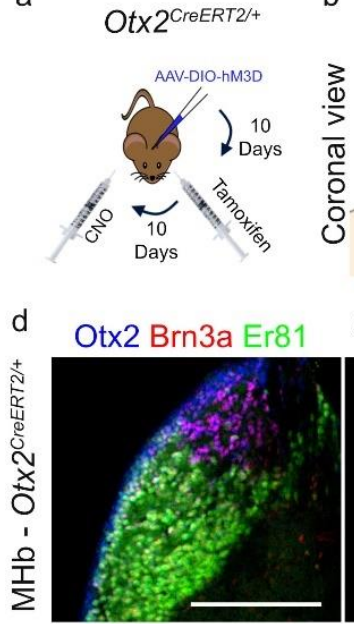

e Otx2

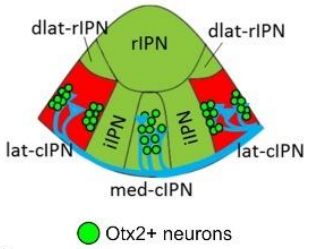

f hM3d Fos
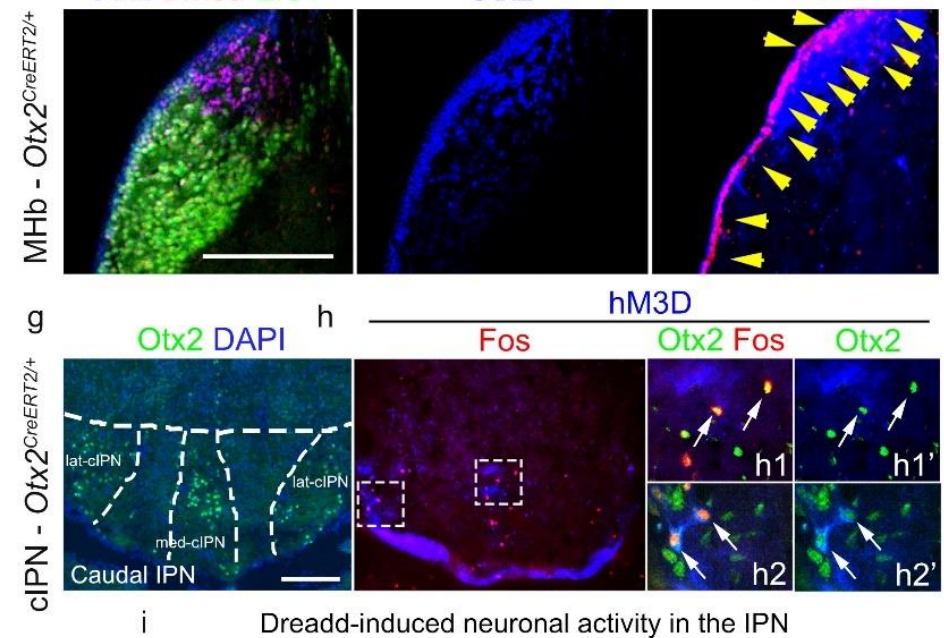

Dreadd-induced neuronal activity in the IPN
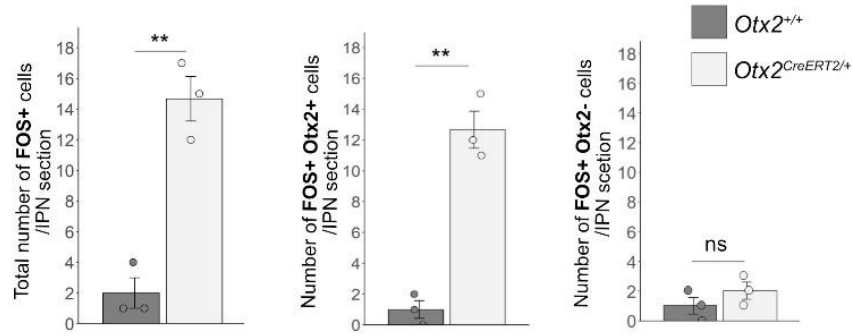

Figure 1. Effects of Otx ${ }^{\mathrm{High}}$ neurons chemoactivation in the MHb. a, Schematic representation of the activating DREADD induction in the Otx $2^{\mathrm{High}}$ neurons of the MHb in $\operatorname{Otx} 2^{\mathrm{CreERT2/+}}$ mice $(\mathrm{n}=3) . \mathbf{b}$, Schematic illustration of an $\mathrm{MHb}$ coronal section representing the localization of substance $\mathrm{P}^{+}$(in red), $\mathrm{ChaT}^{+}$(in green), Otx $2^{\mathrm{High}}$ (in blue) neurons. c, Schematic illustration of an IPN coronal section representing the $\mathrm{O} \times 2^{\mathrm{High}}$ substance $\mathrm{P}$-ergic (in red) and cholinergic (in green) neurons projections and localization of the Otx $2^{+}$neurons of the IPN. d-f, Labelling of MHb coronal sections with anti-Otx2 (in blue), anti-Brn3a (in red), anti-Er81(in green) antibodies (d-e), hM3D-cherry (in blue), anti-Fos antibodies (in red) (f). The yellow arrows point to cells that are co-labeled with hM3D-cherry and antiFos. g-h, Labelling of coronal sections of the IPN with anti-Otx2 antibodies (in green), the nuclear marker DAPI (in blue) (g), anti-Fos antibodies (in red), hM3D-cherry (in blue) (h). h1-h2', Zoom on the med-cIPN (h1,h1') and the lat-cIPN (h2,h2') framed in (h) labeled with anti-Otx2 (in green), hM3Dcherry (in blue) and anti-Fos antibodies (in red). The arrows represent the overlap of hM3D, Fos and Otx2 labelling. i, Quantification of the total number of $\mathrm{Fos}^{+}$cells (left graph), the number of Otx $2^{+} / \mathrm{Fos}^{+}$ cells (middle graph) or the number of Otx $2 / \mathrm{Fos}^{+}$cells in the IPN upon CNO injection in $\operatorname{Otx} 2^{\text {CreERT2/+ }}$ $(\mathrm{n}=3)$ and $\mathrm{Otx}_{2} \mathrm{f}^{++}(\mathrm{n}=3)$ mice. The error bars represent the SEM. $* * \mathrm{P}<0.01$, ns=non significant, bilateral t-test. Scale bar, $100 \mu \mathrm{m}$. 


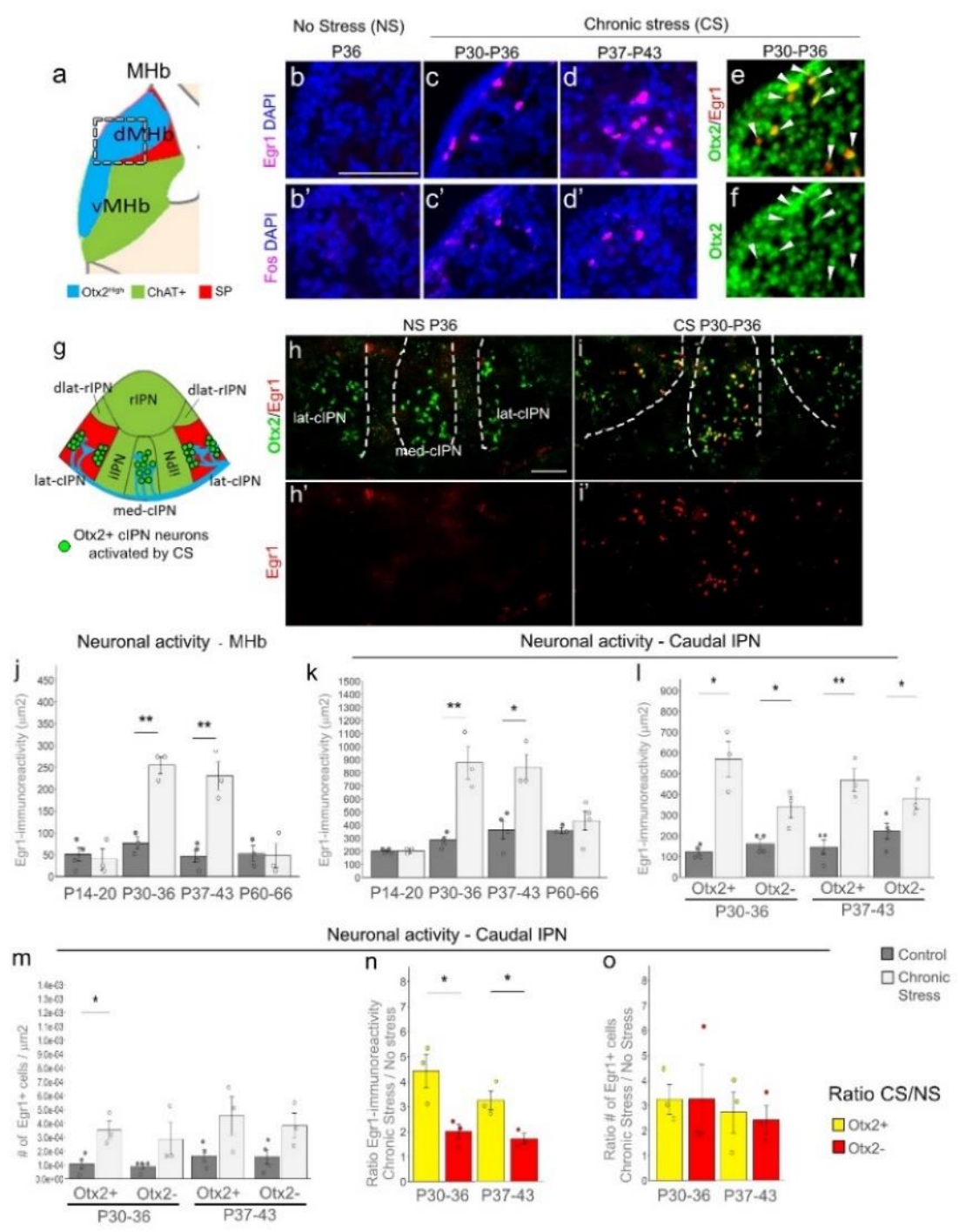

Figure 2. Neuronal activity of the MHb and the IPN in response to CS. a, Schematic representation of an $\mathrm{MHb}$ as described in Fig.1b. b-d', Area corresponding to the frame in (a) labeled with anti-Egr1 (b-d), anti-Fos (b'-d') antibodies and DAPI staining (in blue) in the absence of stress at P36 (b, b'), or in response to a CS between P30-36 (c, $\left.\mathbf{c}^{\prime}\right)$, between P37-43 (d, d'). e-f, Area corresponding to the frame in (a) labeled with anti-Egr1 and anti-Otx2 antibodies in response to a CS between P30-36. White arrowheads show the cells doubly labeled with anti-Egr1 and -Otx2. g, Schematic illustration of the caudal IPN as described in Fig.1c. h-i', Immunolabelling of the caudal IPN coronal sections with antiEgr1 (in red) and anti-Otx2 (in green) antibodies without stress at P36 (h and h') and in response to a CS between P30-36 (i and i'). j-k, Quantification of neuronal activity based on density of Egr1immunoreactivity, after 7 days of stress at different postnatal periods, in the Otx $2^{\text {High }}$ region of the $\mathrm{MHb}$ (CS P14-20 n=3, P30-36 n=3, P37-43 n=3, P60-66 n=3 and no stress P20 n=4, P36 n=3, P43 n=4, P66 $n=3$ ) (j) and in the caudal IPN (CS P14-20 n=3, P30-36 n=3, P37-43 n=3, P60-66 n=4 and no stress P20 $n=4$, P36 n=4, P43 n=4, P66 n=3) (k). l-m, Quantification of neuronal activity based on density of Egr1immunoreactivity colocalizing or not with Otx2 immunoreactivity (l) or based on the number of neurons doubly labeled with anti-Egr1 and anti-Otx2 antibodies (m), after 7 days of stress between P30-36 and P37-43 (CS P30-36 n=3 and no stress P36 n=4; CS P37-43 n=3 and no stress P43 n=4). n-o, ratio between stressed and non-stressed conditions of the same values in 1 and $m$ calculated in $n$ and $o$ respectively. The error bars represent the SEM. $* \mathrm{P}<0.05$, ** $\mathrm{P}<0.01$, bilateral t-test between control 
751 and stressed conditions (j-m), between Otx2 $2^{+}$and Otx2- cells (n-o). Scale bar $50 \mu \mathrm{m}$ b-f (shown in b)., $752100 \mu \mathrm{m} \mathrm{h}$-i' (shown in h).

753 

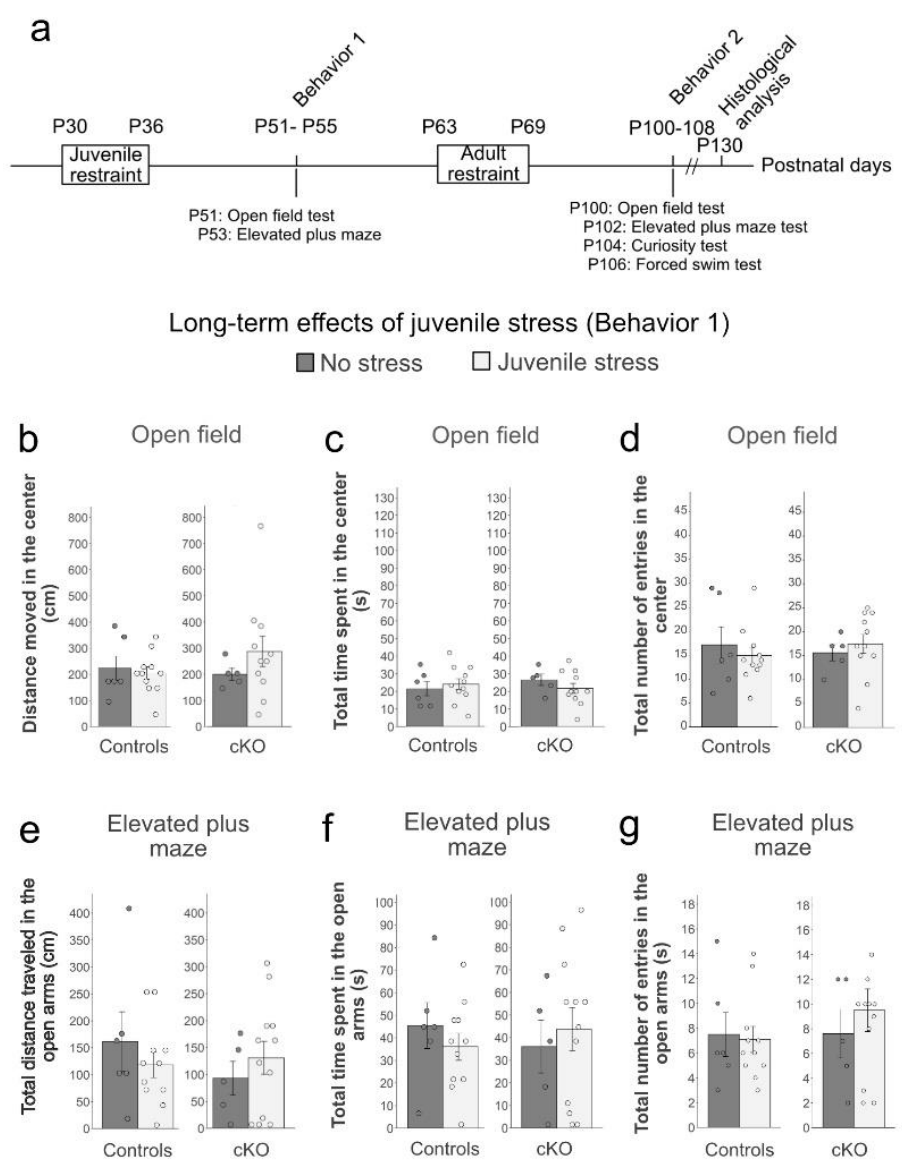

Figure 3. Effects of the pre-adolescent stress on the level of anxiety and depression in the long-term. a, Illustration of the experimental protocol. b-e, Behavioral tests in the open field (b-d) and the elevated plus maze (e-g) on control and Otx2 cKO mice that have not endured stress $(\mathrm{n}=6$ controls and $\mathrm{n}=5$ Otx $2 \mathrm{cKO}$ ) or that have endured a juvenile stress $(\mathrm{n}=11$ controls and 11 Otx $2 \mathrm{cKO}$ for open field, 12 Otx $2 \mathrm{cKO}$ for elevated plus maze) and that have all received tamoxifen injection at P0. Parameters tested include distance traveled $(b, e)$, time spent $(c, f)$, and total number of entries $(\mathrm{d}, \mathrm{g})$ in the center of the open field and in the open arms of the elevated plus maze. The error bars represent the SEM. $* \mathrm{P}<0.05$, bilateral $\mathrm{t}$-test between no stress and stressed conditions. 


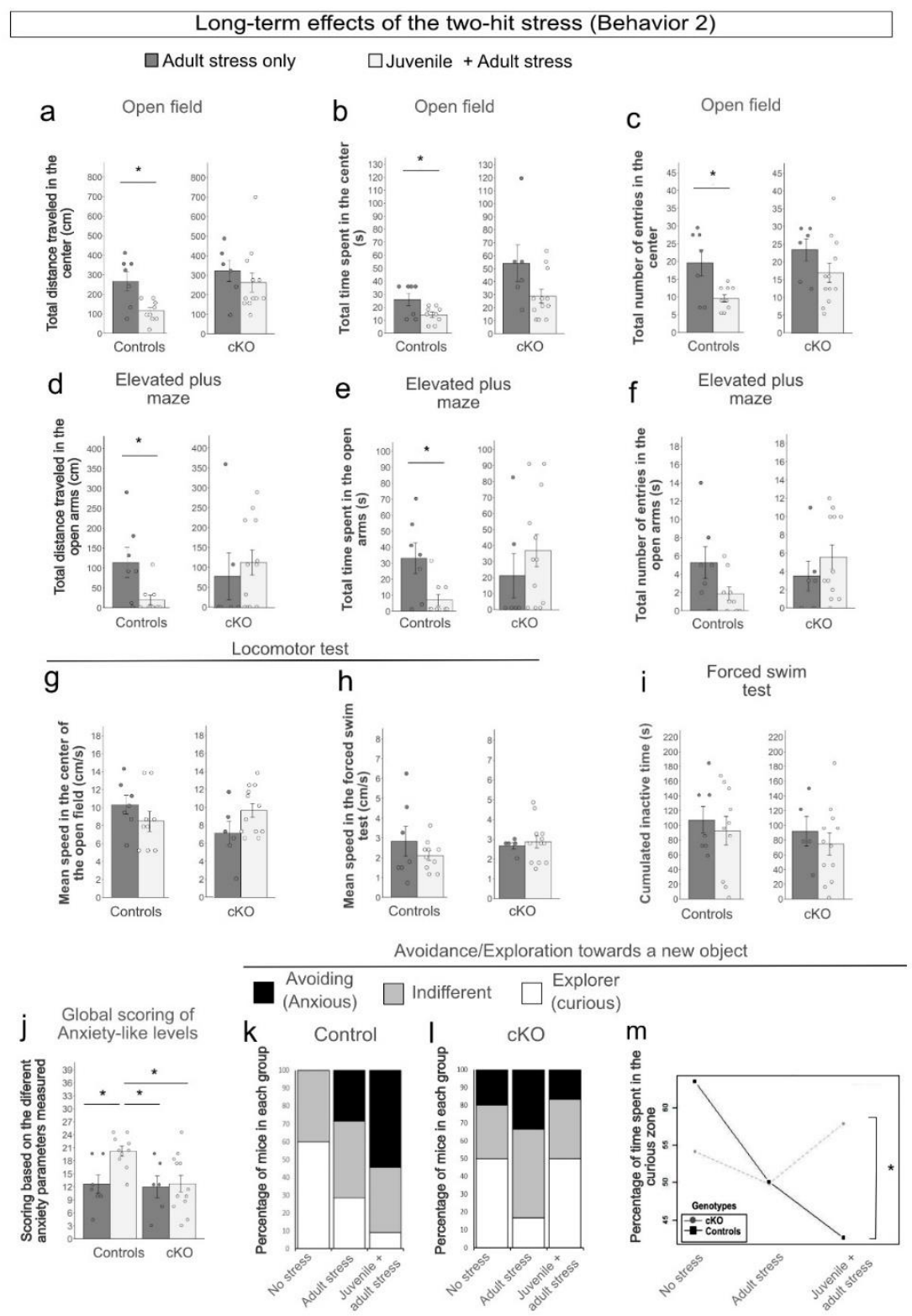

766 Figure 4. Effects of Otx2 deletion alone and in interaction with CS. a-f, Behavioral tests on control and Otx2 $c K O$ that have endured an adult stress but have ( $\mathrm{n}=9$ controls and $\mathrm{n}=12$ Otx $2 \mathrm{cKO}$ ) or have not endured $(\mathrm{n}=7$ controls and $\mathrm{n}=6$ Otx $2 \mathrm{cKO})$ a juvenile stress and that have all received tamoxifen injection at P0. Parameters evaluated include the total distance traveled $(a, d)$ the time spent $(b, e)$ and the number of entries $(c, f)$ in the center of the open field or in the open arms of the elevated plus maze. $\mathbf{g}$-h, Measures of mean speed in the open field and the forced swim test to test for potential motor dysfunctions. i, Time spent inactive in the forced swim test for control and $O t x 2$ cKO mice that have endured an adult stress but have $(\mathrm{n}=10$ controls and $\mathrm{n}=12$ Otx $2 \mathrm{cKO})$ or have not $(\mathrm{n}=7$ controls and $\mathrm{n}=5$ Otx2 $c K O$ ) endured a juvenile stress. $\mathbf{j}$, Global scoring of anxiety based on mean ranking for all the parameters in both open field and elevated plus maze allowing to evaluate anxiety levels in each mouse more accurately. k-l, Percentage of control (k) or Otx2 cKO (l) mice in the exploratory/curious, indifferent and avoiding/anxious subgroup in the avoidance of a novel object test. $\mathbf{m}$. Interaction plot representing the effect of Otx2 deletion and juvenile CS associated with the adult stress on the time spent in the exploratory/curious area in the presence of an object $(F(1,47)=6,713$, Interaction effect 
$\mathrm{p}=0,014)$. The error bars represent the SEM. $* \mathrm{P}<0.05,{ }^{*} \mathrm{P}<0.01$, two-way ANOVA with TukeyHSD post-hoc test $(\mathrm{j}, \mathrm{m})$, bilateral t-test between adult stressed and two-hit stressed conditions (a-i).

782

783 

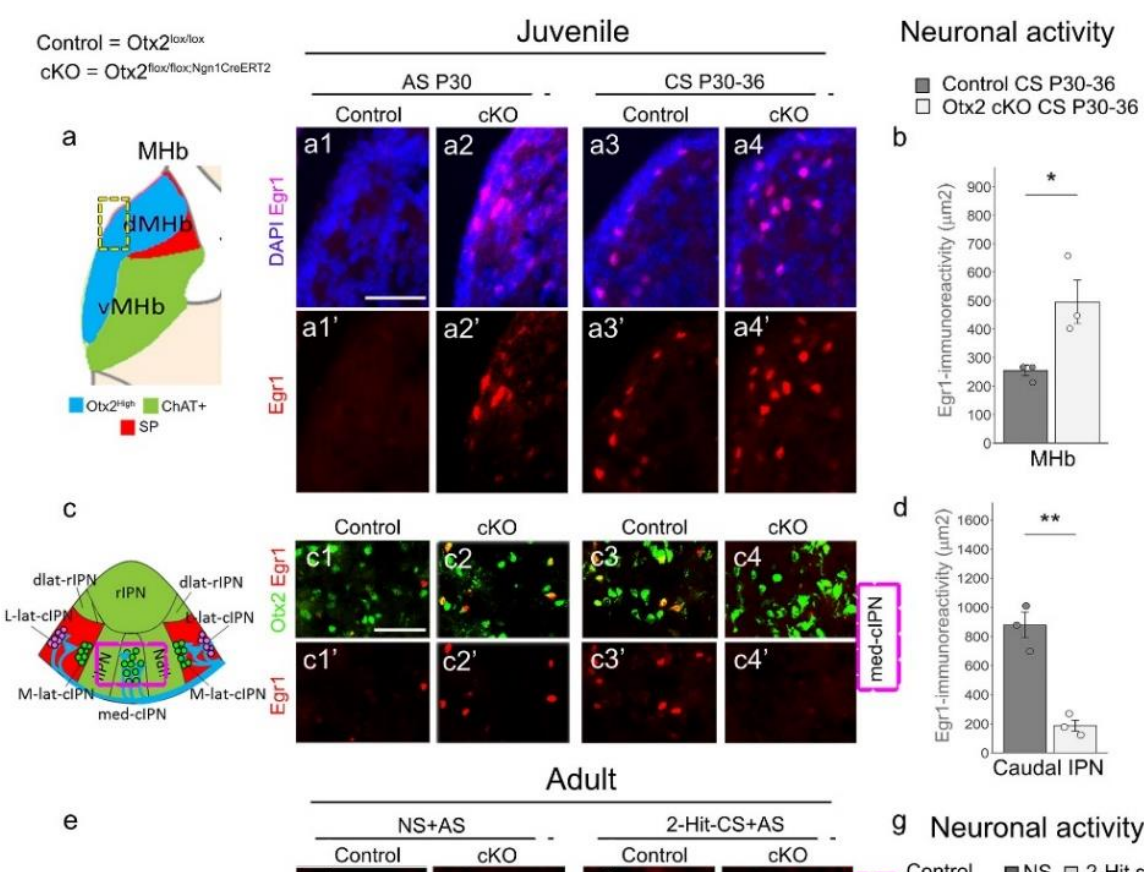

Adult

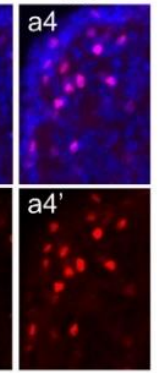

b

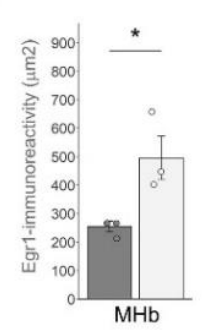

e
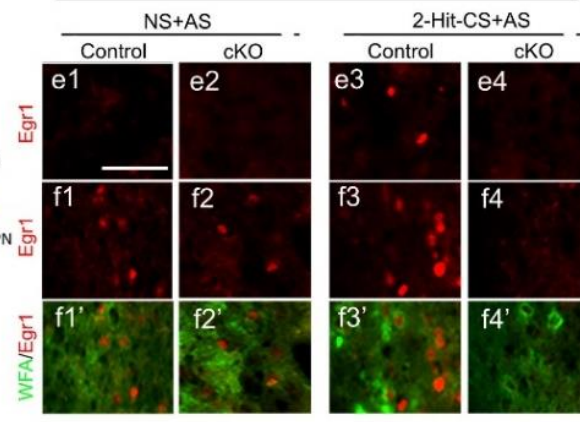

9 Neuronal activity
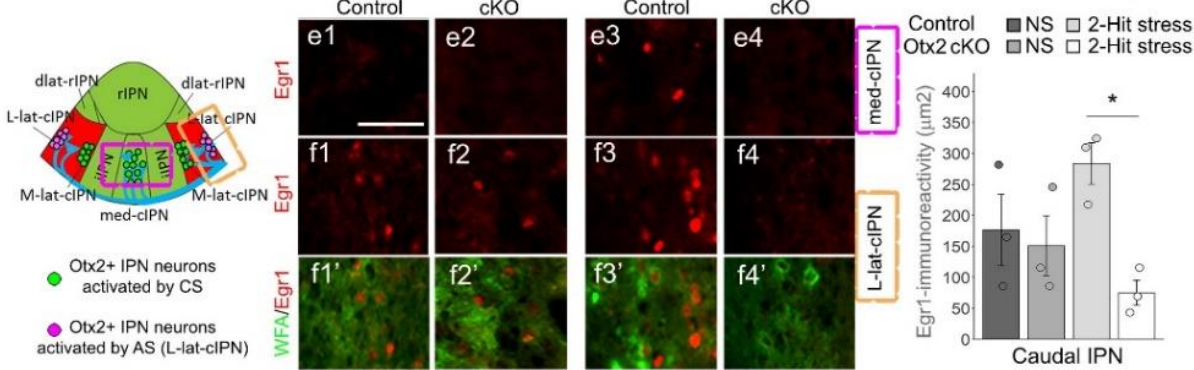

Figure 5. Short- and long-term effects of Otx2 deletion combined to pre-adolescent stress. a,

Schematic representation as described in Fig.1b. a1-a4', Regions framed in (a) of coronal sections stained with anti-Egr1 antibodies (in red) and DAPI (in blue) in control mice ( $\mathrm{n}=3)$ (a1-a1') or in cKO animals $(n=3)\left(a 2-a 2^{\prime}\right)$ that underwent an AS at P30 or in control mice ( $\left.n=3\right)\left(a 3-a 3^{\prime}\right)$ or in cKO animals $(\mathrm{n}=3)(\mathrm{a} 4-\mathrm{a} 4$ ') that underwent a CS between P30-36. $\mathbf{b}$, Quantification of neuronal activity in the MHb based on density of Egr1-immunoreactivity in the animals that underwent a CS between P30-36. c, Schematic illustration as in Fig.1c. c1-c4', Regions framed in (c) from coronal sections labeled with anti-Egr1 (in red) and anti-Otx2 (green) antibodies at the level of the caudal IPN in control mice $(n=3)$ $\left(\mathrm{c} 1-\mathrm{c} 1^{\prime}\right)$ or in cKO animals $(\mathrm{n}=3)\left(\mathrm{c} 2-\mathrm{c} 2^{\prime}\right)$ that underwent an AS at P30 or in control mice $(\mathrm{n}=3)$ (c3c3') or in cKO animals ( $\mathrm{n}=3$ ) (c4-c4') that underwent a CS between P30-36. d, Quantification of neuronal activity in the caudal IPN $b$ ased on density of Egr1-immunoreactivity in the animals that underwent a CS between P30-36. e, Schematic illustration as in Fig.1c. e1-f4', Regions of the medcIPN framed in (d) (e1-e4) or of the L-lat-cIPN framed in (d) (f1-f4') from coronal sections labeled with anti-Egr1 (in red) and anti-WFA (green) antibodies at the level of the caudal IPN in controls ( $n=3$ ) (e1-f1') and in cKO mice (n=3) (e2-f2') that underwent a final AS only or a 2-hit stress protocol ${ }^{+} \mathrm{a}$ final AS in controls $(\mathrm{n}=3)(\mathrm{e}-\mathrm{f} 3$ ') and in $\mathrm{cKO}$ mice $(\mathrm{n}=3)$ ( $(\mathrm{e}-\mathrm{f} 4$ '). $\mathrm{g}$, Quantification of neuronal activity in the caudal IPN based on the density of Egr1-immunoreactivity after a final AS before sacrifice in the caudal IPN of controls and Otx2 cKO that have or have not endured the two-hit stress protocol. The error bar represents the SEM. $* \mathrm{P}<0.05, * * \mathrm{P}<0.01$, bilateral $\mathrm{t}$-test $(\mathrm{b}, \mathrm{d})$, two-way ANOVA with TukeyHSD post-hoc test (g). Scale bar $25 \mu \mathrm{m}$ a1-a4' (shown in a1)., $50 \mu \mathrm{m} \mathrm{c1-c4'(shown} \mathrm{in} \mathrm{c1),} \mathrm{e1-}$ f4'(shown in e1). 

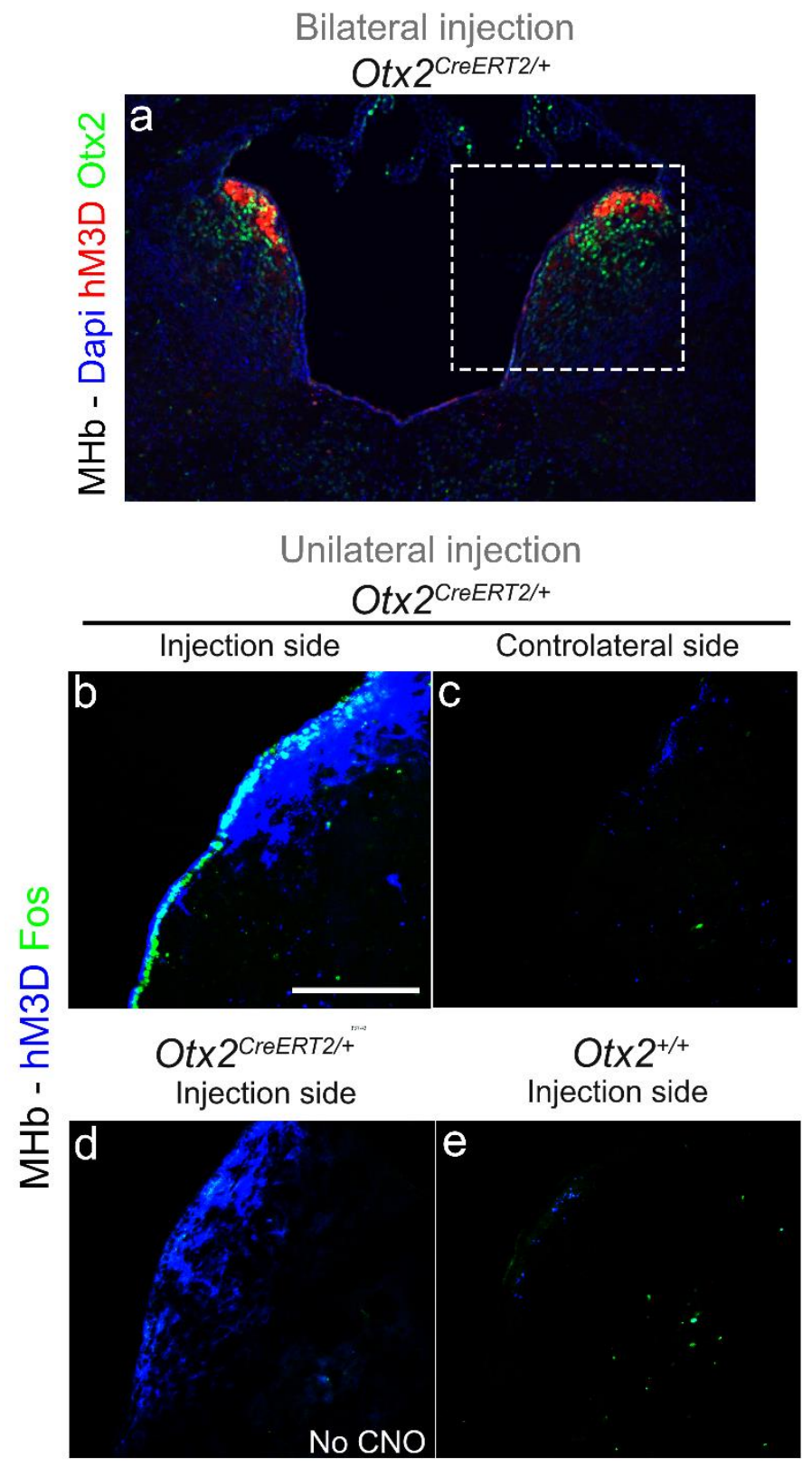

811 Supplemental figure S1. Control experiments of Otx $2^{\text {High }}$ neurons chemoactivation in the MHb.

812 a, coronal section at the level of the MHb labeled with hM3D-cherry (in red) and anti-Otx2 antibodies 813 (in green), in mice stereotaxically injected in the bilateral MHb with viruses expressing the DREADDs. 814 b-e, labelling in the equivalent region framed in (a) with hM3D-cherry (in blue) and anti-Fos antibodies 815 (in green), in mice stereotaxically injected in one side of the MHb with viruses expressing the 816 DREADDs. The injected side (a; same image as Fig.1f) and the controlateral side of the injection (b) in 817 Otx $2^{\text {CreERT2/+ }}$ mice that have been injected with CNO $(n=3)$ are shown. The injected side (c) in 818 Otx $2^{\text {CreERT2/+ }}$ mice that did not receive CNO $(n=3)$ is shown. The injected side in wild type mice that 819 were injected with CNO (n=3) is shown. Scale bar : $100 \mu \mathrm{m}$. 
820

821

822

823

824

825

826

827

828

829

830

831

832

833 Supplemental figure S2. Scheme comparing the different stages of a human life and the mice 834 equivalent age.

\section{$\underline{\text { Human life stages }}$}

\section{Pre-Adolescence}

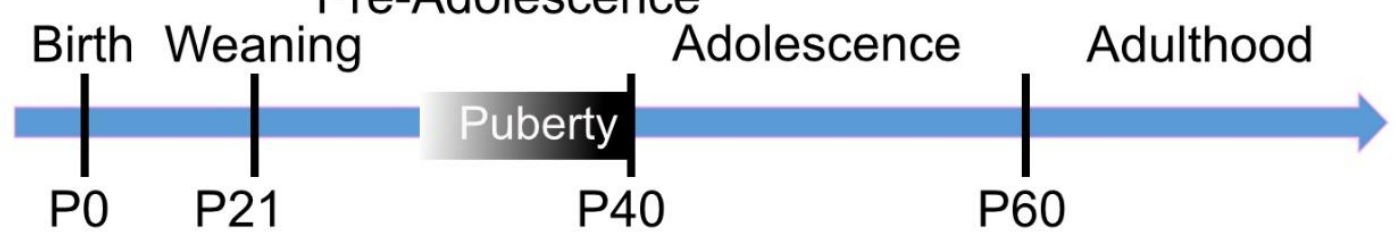

Equivalent mice ages

835 


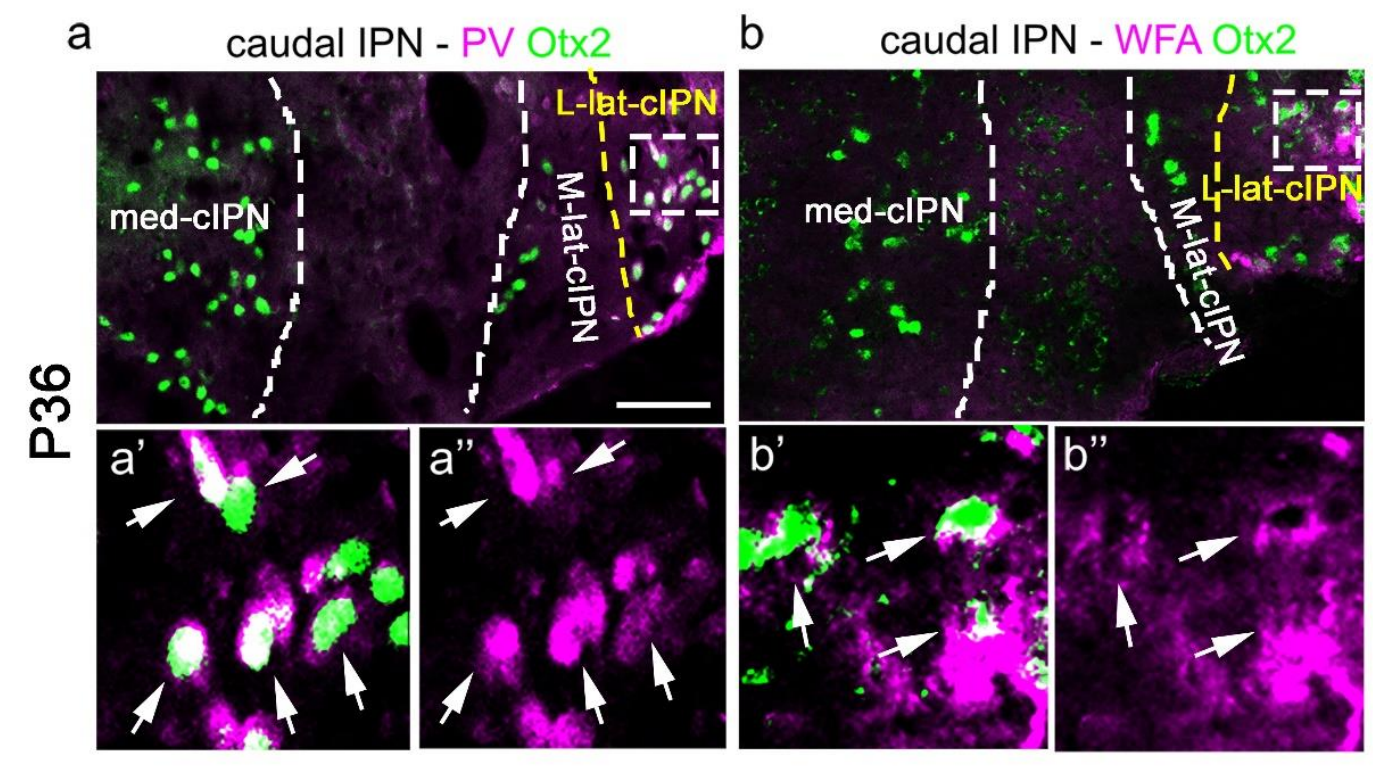

838 Supplemental figure S3. Molecular signature of the Otx2 ${ }^{+}$neurons in the lateral and central 839 subnuclei of the IPN. a, Immunolabelling of IPN coronal sections with anti-PV (in red) and anti-Otx2 840 (in green) antibodies at P36 (n=5). a'-a', Zoom on the area framed on (a). The white arrows show the 841 colocalization of the immunoreactivities for PV and Otx2. b, Labelling of the IPN coronal sections with 842 WFA, binding to PNNs, (in red) and anti-Otx2 antibodies (in green) at P36. b'-b', Zoom on the area 843 framed on (b). The white arrows show the colocalization of WFA and anti-Otx2 antibodies. Scale bar $84450 \mu \mathrm{m}$. 


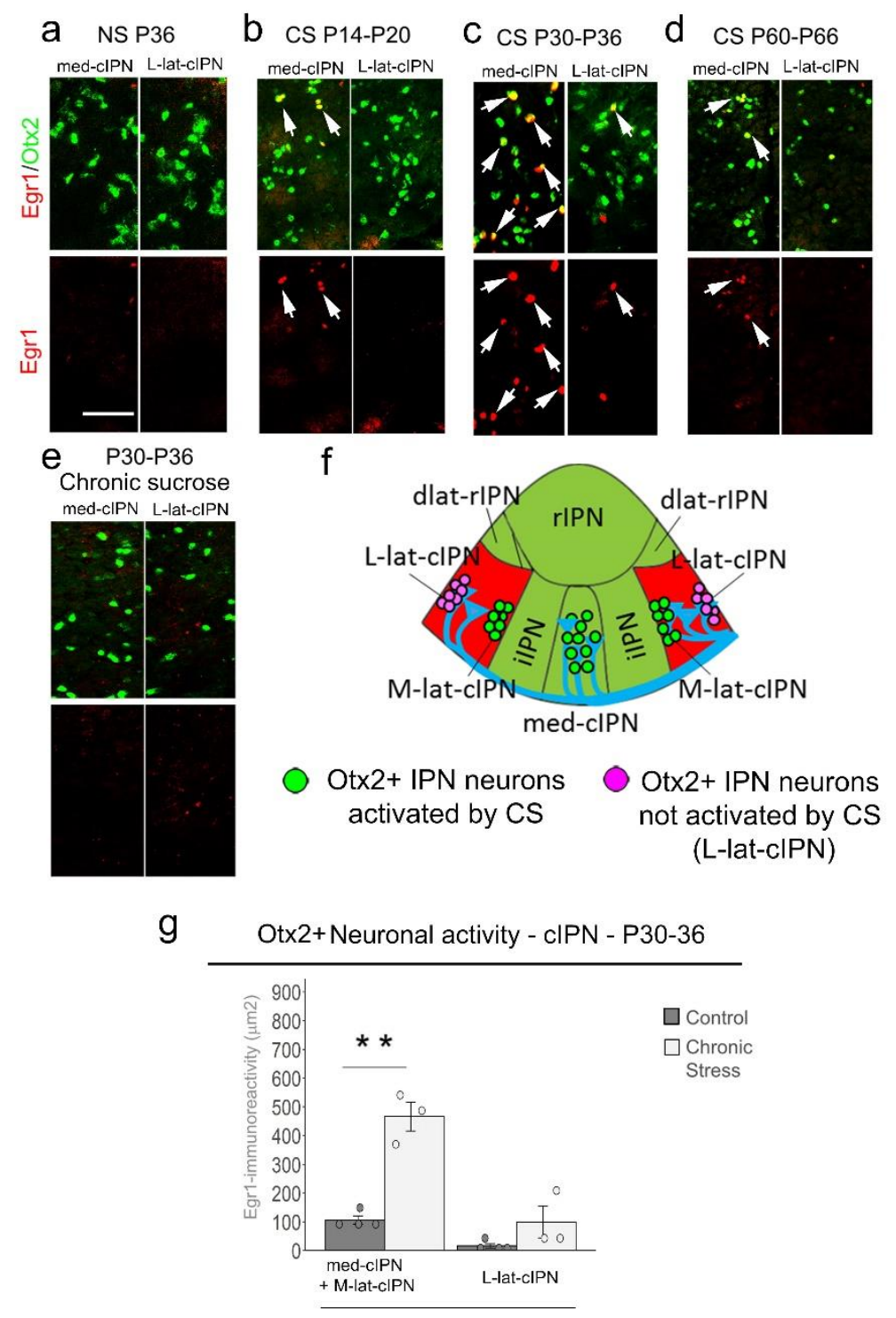

848 Supplemental figure S4. Neuronal activity in med-cIPN+M-latcIPN and L-lat-cIPN in response 849 to chronic stress at different developmental periods. a-f, Regions of the med-cIPN and L-latcIPN 850 in coronal sections at the level of the IPN labeled with anti-Egr1 and anti-Otx2 from mice that endured 851 no stress at P36 (a), CS between P14-20 (n=3)(b), CS between P30-36 ( $\mathrm{n}=3)(\mathrm{c})$, CS between P60-66 $852(\mathrm{n}=4)(\mathrm{d})$, or chronic sucrose consumption between P30-36 $(\mathrm{n}=3)(\mathrm{e})$. f, Schematic illustration of the 853 caudal IPN regions in coronal sections where Otx $2^{\text {High }}$ substance P-ergic (in red) and cholinergic (in 854 green) neurons of the MHb project and localization of the Otx2 $2^{+}$neurons of the IPN. g, Quantification 855 of Egr1-immunoreactivity density in med-cIPN+M-lat-cIPN and in L-lat-cIPN regions in Otx2+ cells 856 in mice that have endured NS $(n=4)$ or $C S(n=3)$. The error bar represents the SEM. $* \mathrm{P}<0.05$, $* * P<$ 857 0.01, bilateral t-test between control (no stress) and stressed conditions. Scale bar : $25 \mu \mathrm{m}$ 
a

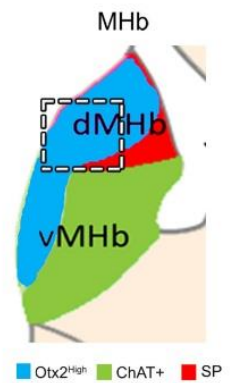

d

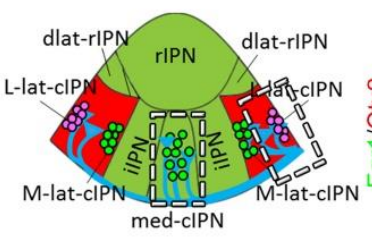

Otx2+ IPN neurons activated by CS

Otx2+ IPN neurons activated by AS (L-lat-clPN)

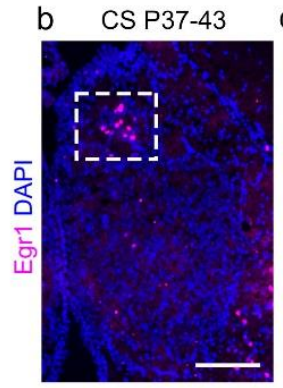

e CS P37-43

med-cIPN L-lat-cIPN

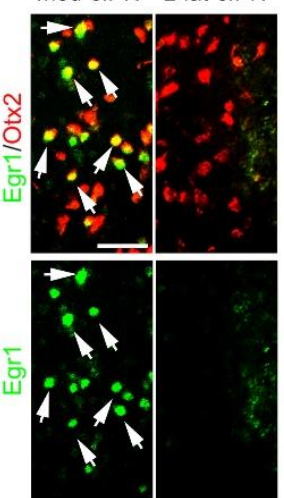

f Acute stress P43 med-cIPN L-lat-cIPN

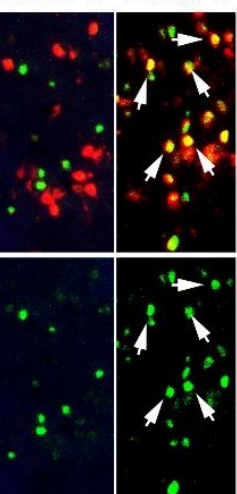

Neuronal activity - Caudal IPN

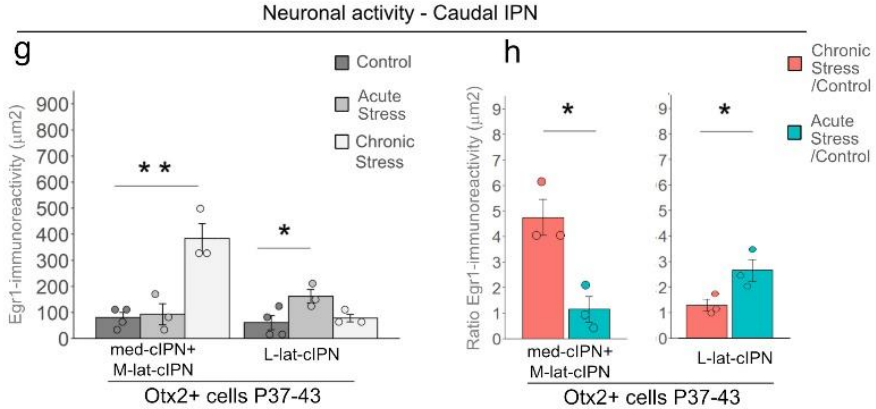
acute stress in juvenile mice. a, Schematic representation of an $\mathrm{MHb}$ coronal section showing the localization of substance $\mathrm{P}+$ (in red), ChAT+ (in green), Otx $2^{\text {High }}$ (in blue) neurons. b-c, Coronal sections at the level of the $\mathrm{MHb}$ with the same region as in (a) showed. Labeling with ant-Egr1 antibodies and DAPI staining (in blue) in response to a chronic stress between P37-43 (n=3) (b) or to an acute stress at $\mathrm{P} 43$ (n=3) (c). The approximate same region is framed in (a), (b) and (c). d, Schematic illustration of the caudal IPN regions in coronal sections where Otx $2^{\text {High }}$ substance P-ergic (in red) and cholinergic (in green) neurons of the MHb project and localization of the Otx $2^{+}$neurons of the IPN. ef, Coronal sections at the level of the IPN with the same regions framed in (d) of the med-cIPN and Llat-cIPN showed. Immunolabeling with anti-Egr1 (in red) and anti-Otx2 (in green) antibodies in response to a chronic stress between P37-43 (n=3) (e) or to an acute stress at P43 (n=3) (f). g, Quantification of Egr1-immunoreactivity density in Otx2+ cells between in the med-cIPN+M-lat-cIPN and the L-lat-cIPN at P43 upon AS $(n=3), C S(n=3)$, or NS $(n=4)$. $\mathbf{h}$, same measurements shown in $g$ for AS and CS relative to the mean NS value. The error bar represents the SEM. $* \mathrm{P}<0.05, * * \mathrm{P}<0.01$, bilateral t-test between chronic stress and acute stress or no stress conditions. Scale bar $50 \mu \mathrm{m} \mathrm{b}-\mathrm{c}$ (shown in b), $25 \mu \mathrm{m}$ e-f (shown in e). 

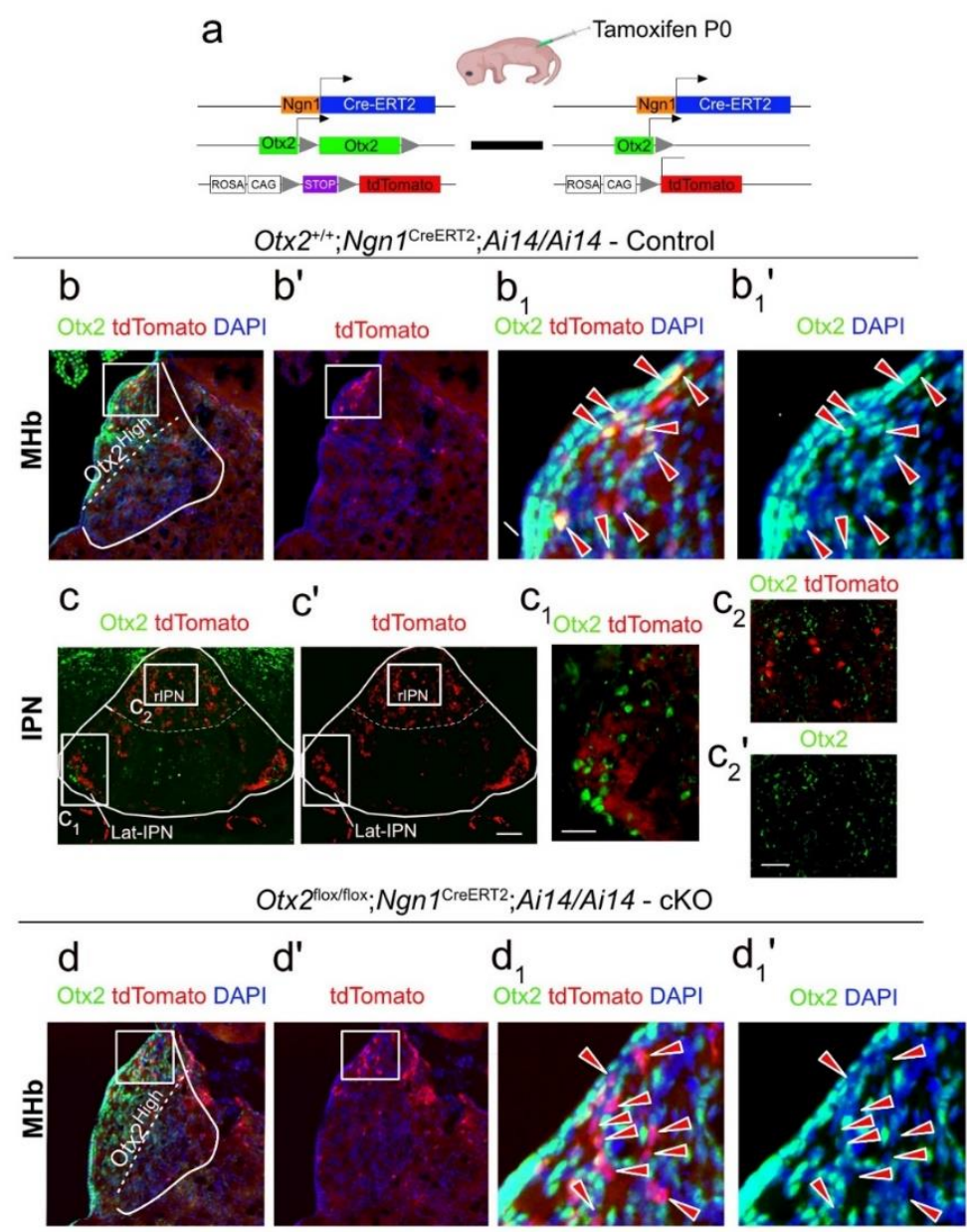

Otx $2^{\text {flox/flox; } N g n 1^{\text {CreERT2}} ; A i 14 / A i 14 \text { - }}$ CKO

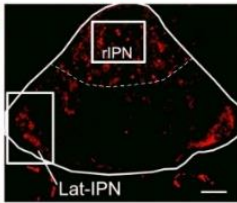

d'
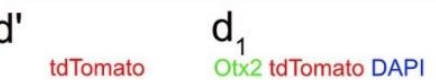

$\mathrm{d}_{1}^{\prime}$

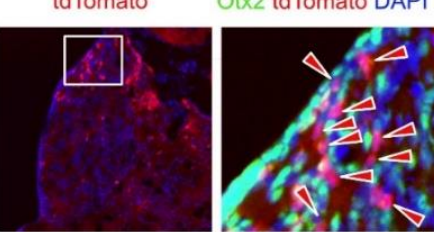

Otx2 DAPI
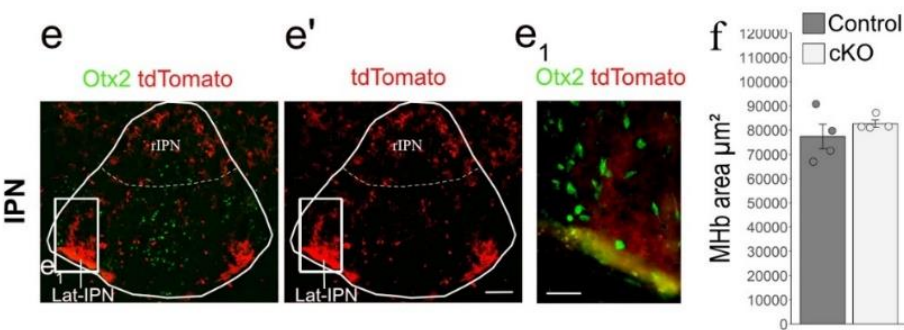

878 Supplemental figure S6. Expression of tdTomato and effects of Otx2 deletion on the HIPS. a, 879 Schematic representing the strategy used to induce the recombination of Otx2 and $880 \mathrm{Gt}(\mathrm{ROSA}) 26 \mathrm{Sor}(\mathrm{tdT}$ Tomato-WPRE) floxed alleles specifically in MHb neurons observed at P30. b-c, Labelling of coronal sections at the level of the MHb (b-b') and the IPN (c-c') in control mice ( $\mathrm{n}=3$ ) with DAPI staining (in blue), anti-Otx2 antibodies (in green) and tdTomato (in red). Arrowheads in b and b' point to tdTomato ${ }^{+}$cells that are not Otx2+, ressembling glial cells. $\mathbf{b}_{\mathbf{1}}-\mathbf{b}_{\mathbf{1}}$ ', Zoom on the area framed in $b$ and $b$ '. The arrowheads in $b_{1}$ and $b_{1}$ ' point to cells co-expressing Otx2 and tdTomato. $\mathbf{c}_{1}$, Zoom on the area of Lat-IPN framed in c and c'. $\mathbf{c}_{2}-\mathbf{c}_{2}$ ', Zoom on the area of rIPN framed in $c$ and c'. d-e, Labelling of coronal sections with anti-Otx2 antibodies (in green), tdTomato (in red) and DAPI staining (in blue) at the level of the MHb (d-d') and the IPN (e-e') in Otx2 cKO mice (n=3). $\mathbf{d}_{\mathbf{1}}$-d $\mathbf{d}_{\mathbf{1}}$ ', Zoom on the area framed in $d$ and $d$ '. The arrowheads in $d_{1}$ and $d_{1}$ ' point to cells recombined and do not present Otx2-immunoractivity. $\mathbf{e}_{1}$, Zoom on the area framed in e and e'. $\mathbf{f}$, Quantification of the total surface of the $\mathrm{MHb}$ in $\mu \mathrm{m}^{2}$ in controls $(\mathrm{n}=3)$ and in Otx $2 \mathrm{cKO}$ mice $(\mathrm{n}=3)$. The error bar represents the

891 SEM. $P$ is not significant, bilateral t-test. Scale bars: b-e' $=100 \mu \mathrm{m}$ (shown in b', c', d', and e'); $c_{1}, e_{1}$ $=50 \mu \mathrm{m} ; \mathrm{b} 1, \mathrm{~b} 1^{\prime}, \mathrm{c} 2, \mathrm{c} 2^{\prime}, \mathrm{d} 1, \mathrm{~d} 1^{\prime}=25 \mu \mathrm{m}$ (shown in b1', c2'). 

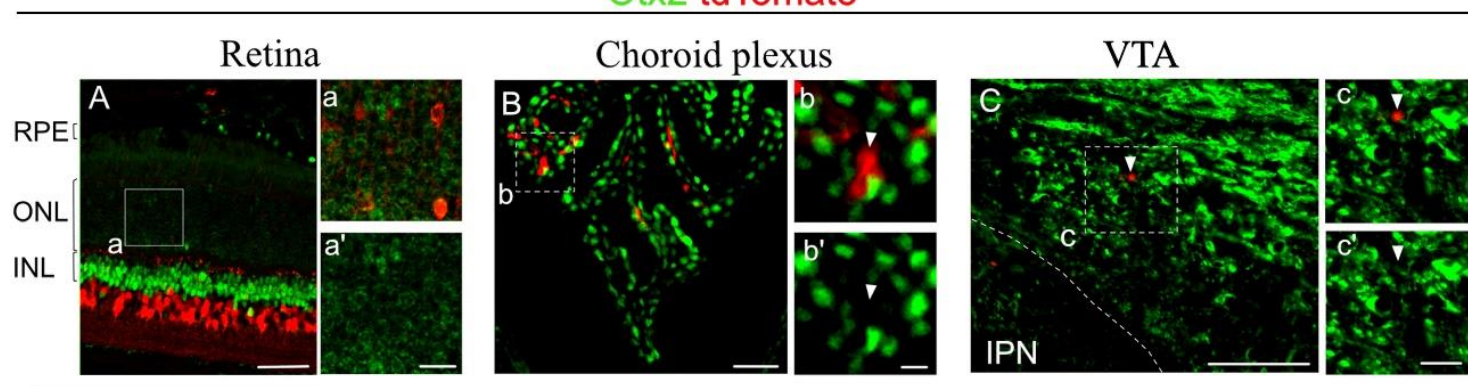

906

907

908 Supplemental figure S7. Regions expressing Otx2 and TdTomato in $\mathrm{Otx2}^{+/+} ; \mathrm{NgnICre}^{E R T 2}$

909 ;Ai14/Ai14 mice. a-f, Labelling with anti- Otx2 antibodies (in green) and tdTomato (in red) of retina 910 sections (a), coronal section of the choroid plexus (b), VTA (c), colliculi (d), septum (e) and LGN (f).

911 a1-a1', b1-b1', c1-c1', d1-d1' Zoom on the area framed in a, b, c, and d, respectively. RPE: retinal 912 pigmented epithelium. ONL: outer nuclear layer containing photoreceptors. INL: inner nuclear layer 913 containing bipolar cells. LSD: lateral septum dorsal part. LSI: lateral septum intermediate part. LSV: 914 lateral septum ventral part. LGN: lateral geniculate nucleus. Scale bars: $\mathrm{a}-\mathrm{f}=100 \mu \mathrm{m}$; $\mathrm{a} 1-\mathrm{d} 1$ ' $=25 \mu \mathrm{m}$ 915 (shown in al'-d1'). 


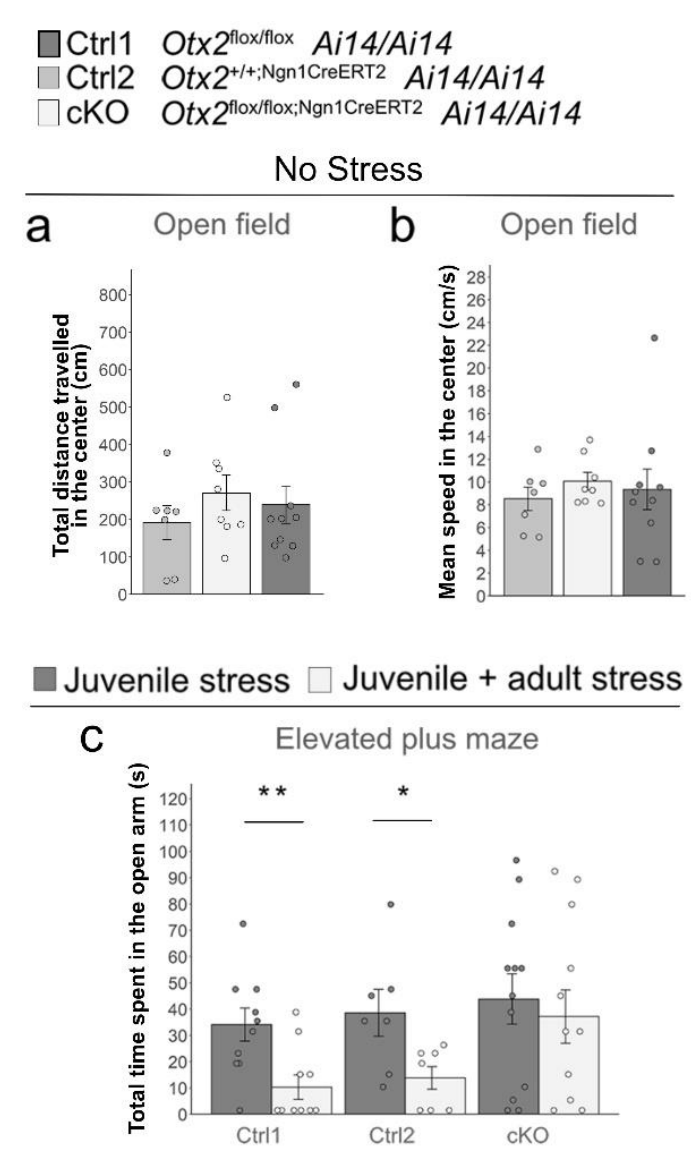

918 Supplemental figure S8. Control for the effect of Ngn1CreERT2 genetic background in normal 919 and stressed conditions on behavior. a-b, Behavioral tests on Otx2lox/lox ;Ai14/Ai14 ( $\mathrm{n}=10)$, 920 Otx2+/+;Ngn1CreERT2 ;Ai14/Ai14 $(n=7)$ and Otx2 cKO $(n=8)$ measuring the total distance traveled 921 (a) and the mean speed (b) in the open field. These animal groups have not endured any previous stress. 922 c, Behavioral tests evaluating anxiety-like phenotype by measuring the total time spent in the open arms 923 of the elevated plus maze for Ctrl1, Ctrl2 and Otx 2 cKO mice that have endured juvenile ( $\mathrm{n}=10,7,12$, 924626 respectively) or juveline + adult stresses ( $n=10,7,11$, respectively). The error bar represents the 925 SEM. $* \mathrm{P}<0.05$, **P $<0.01$, One-way ANOVA $(\mathrm{a}, \mathrm{b})$, bilateral $\mathrm{t}$-test between juvenile stress and two926 hit stress conditions (c). 


\section{Acute restraint stress Egr1 Dapi}

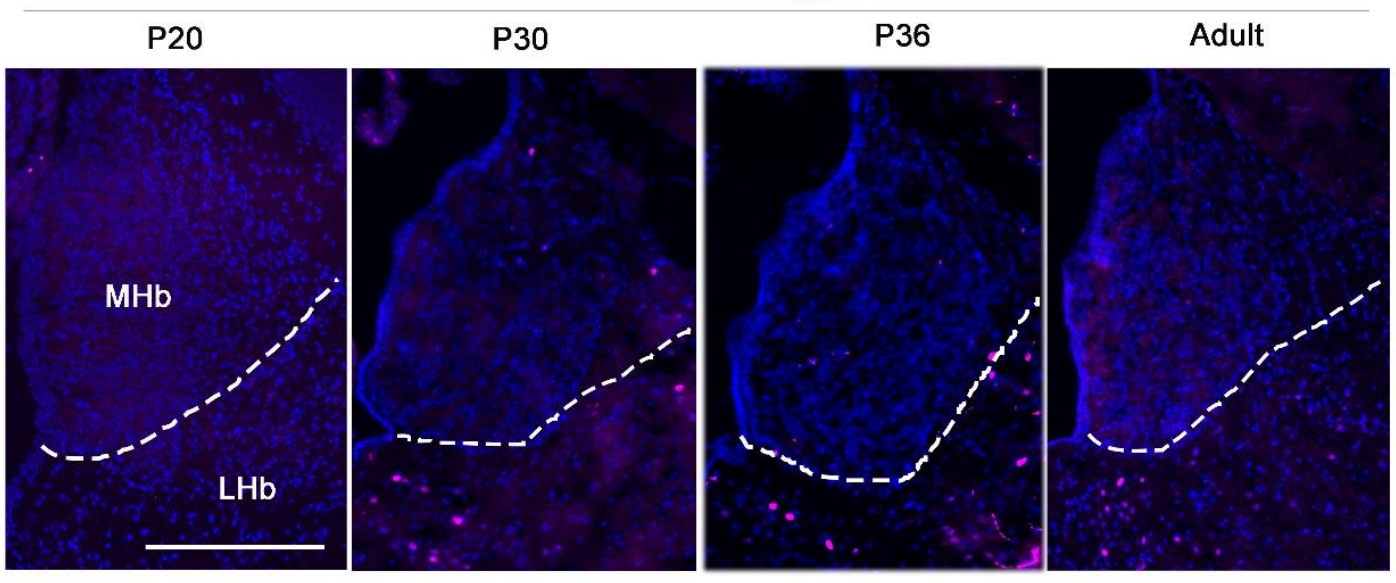

930 Supplemental figure S9. Effect of acute stress on neuronal activation in the MHb. Coronal sections 931 at the level of the MHb stained with anti-Egr1 antibodies (in magenta) and DAPI staining (in blue) from 932 brains collected at different developmental stages (from P20 to Adulthood) after an acute restraint 933 stress. (P20 n=3; P30 n=3; P36 n=5; Adult n=4). Scale bar : $100 \mu \mathrm{m}$. 


\section{Adult - Egr1}
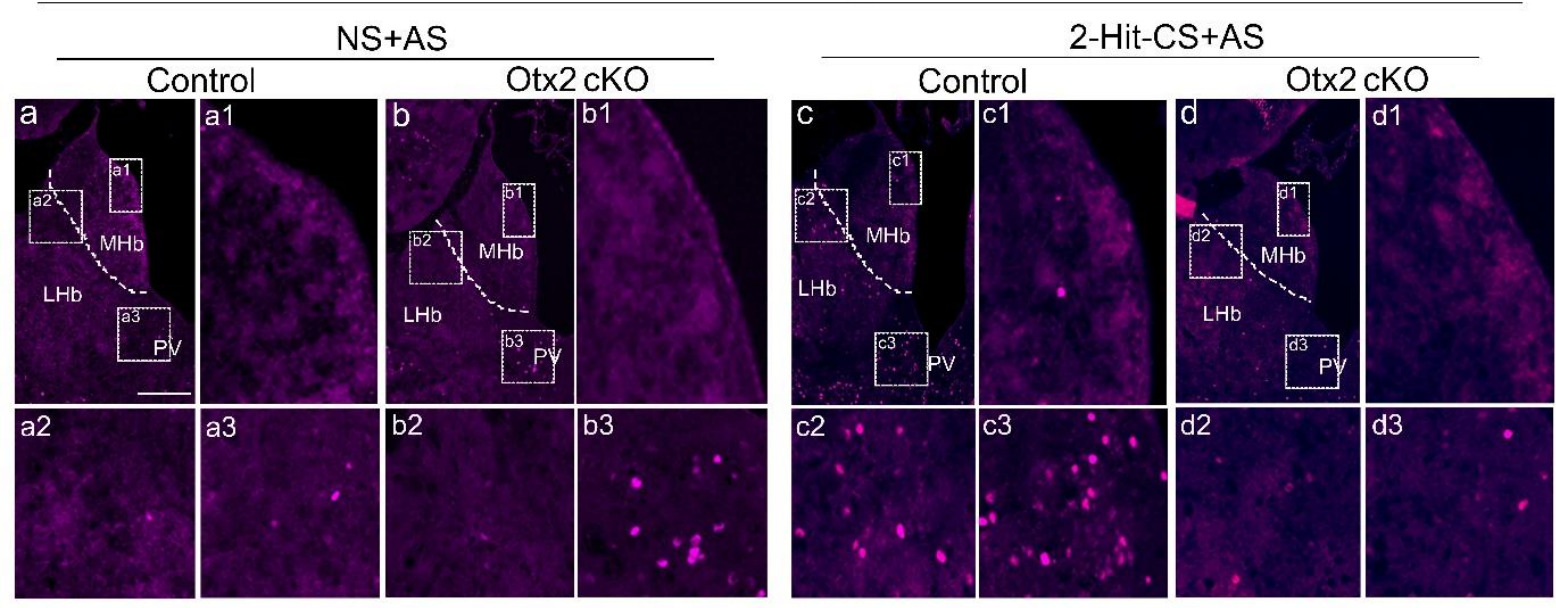

935

936 Supplemental figure S10. Effects of Otx2 deletion in the dMHb on the neuronal activity of the

937 habenula and other stress-related regions in the long-term in response to a last acute stress. a-d,

938 Labelling of coronal sections with anti-Egr1 antibodies (in magenta) at the level of the MHb in control

$939(\mathrm{n}=3)(\mathrm{a})$ and in Otx2 cKO (n=3) (b) animals that only endured an acute stress before the sacrifice or

940 after the "two-hit stress" protocol (juvenile+adult chronic restraint stress) followed by a last acute stress

941 in controls $(\mathrm{n}=3)(\mathrm{c})$ and Otx2 cKO mice $(\mathrm{n}=3)(\mathrm{d})$. a1-a3, b1-b3, c1-c3, d1-d3, Zoom on the area framed

942 in a, b, c, d, respectively. LHb = Lateral Habenula, PV = Paraventricular nucleus of the thalamus. Scale

943 bar: $100 \mu \mathrm{m}$ in a, b, c, d (shown in a). 
Supplemental figure S11. Proposed mechanism of susceptibility or resilience to-stress inducing anxiety taking roots during the peripubertal period. The maturation of the stress response in HIPOPS circuits is taking place during the prepubertal period. In control situation, no chronic stresses are endured, and the maturation is normal in the presence or absence of $O t \times 2$ (green curve). If a chronic stress is experienced during the critical period, the activity is higher by the time of closure (red curve). In the absence of Otx 2 expression in the $\mathrm{MHb}$, a chronic stress activates even more strongly the $\mathrm{MHb}$, leading to abnormally low response of the IPN by the time of critical period closure (blue curve). In all cases, following closure of the critical period, the IPN activity in response to new stresses during later life is dependent on the activity it had at the end of the critical period. This activity reflects the 
972 Supplemental table S1 (See file in Excel format)

973

974

975

976

977

978

979 


\section{Supplementary Files}

This is a list of supplementary files associated with this preprint. Click to download.

- TableS1ResearchsquareV4.xlsx 